\title{
Mode competition in a system of two parametrically driven pendulums; the dissipative case
}

\author{
E. J. Banning ${ }^{1}$, J. P. van der Weele*, J. C. Ross ${ }^{2}$, M. M. Kettenis ${ }^{2}$, E. de Kleine ${ }^{3}$ \\ Center for Theoretical Physics, University of Twente, P.O. Box 217, 7500 AE Enschede. The Netherlands
}

Received 21 February 1997

\begin{abstract}
In this paper we study the dynamics of a system of two linearly coupled, parametrically driven pendulums, subject to viscous dissipation. It is a continuation of the previous paper (E.J. Banning and J.P. van der Weele (1995)), in which we treated the Hamiltonian case. The damping has several important consequences. For instance, the driving amplitude now has to exceed a threshold value in order to excite non-trivial motion in the system. Furthermore, dissipative systems (can) exhibit attraction in phase space, making limit cycles, Arnol'd tongues and chaotic attractors a distinct possibility. We discuss these features in detail. Another consequence of the dissipation is that it breaks the time-reversal symmetry of the system. This means that several, formerly distinct motions now fall within the same symmetry class and may for instance annihilate each other in a saddle-node bifurcation. Implications of this are encountered throughout the paper, and we shall pay special attention to its effect on the interaction between two of the normal modes of the system.
\end{abstract}

Keywords: Dissipation; Limit cycles; Arnol'd tongues; Symmetry breaking; Coupled pendulums

\section{Introduction}

\subsection{The system and its motions}

In the previous paper [1] we studied a Hamiltonian system of two identical parametrically driven pendulums, coupled by a linear torsion spring. This provided us with a good idea of the dynamical structure, but since in practical situations (some)

\footnotetext{
* Corresponding author.

${ }^{1}$ Current address: EPT-RF, Shell International Exploration and Production B.V., Volmerlaan 8, 2280 AB Rijswijk, The Netherlands.

${ }^{2}$ Current address: Institute for Theoretical Physics, University of Amsterdam, Valckenierstraat 65. 1018 XE Amsterdam, Netherlands.

${ }^{3}$ Current address: ENT Department, Audiology, University Hospital, P.O. Box 30.001, 9700 RB Groningen, Netherlands.
} 


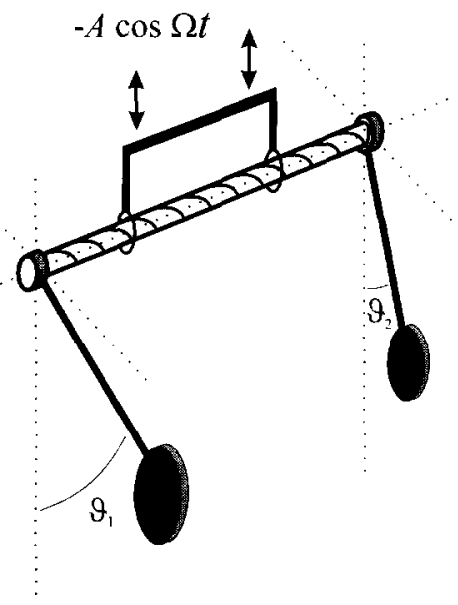

Fig. 1. The system, consisting of two parametrically driven pendulums, coupled by a linear torsion spring.

dissipation is always present, we had yet to include this into our model to make contact with experiments. Now, the dissipative forces in a pendulum system will, in general, include various terms: a term which depends solely on the sign of the velocity, one which is linear in the velocity, one quadratic in the velocity, and perhaps even terms of higher order [2]. Our main interest, however, is not to model any specific dissipative force, but to study the generic effect of dissipation on the dynamics of the system and, in particular, on the phenomenon of mode competition. We will therefore keep our equations of motion as simple as possible and adopt a dissipation which is linear in the angular velocity of the pendulums, the so-called viscous damping. This kind of damping is the one generally used by other authors as well, and this choice therefore has the additional advantage that it facilitates a comparison with other theoretical work, in particular with that of Skeldon et al. $[3,4]$.

Fig. 1 depicts the system, and the equations of motion are readily written down:

$$
\begin{aligned}
& \ddot{\vartheta}_{1}+f(t) \sin \vartheta_{1}+\gamma \dot{\vartheta}_{1}+K\left(\vartheta_{1}-\vartheta_{2}\right)=0, \\
& \ddot{\vartheta}_{2}+f(t) \sin \vartheta_{2}+\gamma \dot{\vartheta}_{2}-K\left(\vartheta_{1}-\vartheta_{2}\right)=0 .
\end{aligned}
$$

Here the damping is represented by the $\gamma$ terms $^{3}$, and the remaining parameters are as before: the coupling parameter $K$, given by

$$
K \equiv \frac{C}{m l^{2}},
$$

\footnotetext{
${ }^{3}$ Note that this choice of dissipation corresponds to viscous damping in the pivots and is not a good model for the resistance due to the pendulums' motion through the air. A realistic model for the air friction would involve a non-angular (but vertical) component, since the whole system is being moved up and down.
} 
where $C$ is the torsion-spring constant, and the function $f(t)$, which contains the driving force,

$$
f(t)=\frac{1}{l}\left(g+A \Omega^{2} \cos \Omega t\right) .
$$

In the numerical calculations we will always take the length of the pendulums to be $l=1 \mathrm{~m}$.

We sometimes find it convenient to work with normal coordinates, given by

$$
\begin{aligned}
& \phi_{1} \equiv \vartheta_{1}+\vartheta_{2}, \\
& \phi_{2} \equiv \vartheta_{1}-\vartheta_{2} .
\end{aligned}
$$

The equations of motion then become

$$
\begin{aligned}
& \ddot{\phi}_{1}+2 f(t) \sin \frac{1}{2} \phi_{1} \cos \frac{1}{2} \phi_{2}+\gamma \dot{\phi}_{1}=0, \\
& \ddot{\phi}_{2}+2 f(t) \sin \frac{1}{2} \phi_{2} \cos \frac{1}{2} \phi_{1}+\dot{\phi}_{2}+2 K \phi_{2}=0 .
\end{aligned}
$$

We study the dynamics of the system by means of its periodic orbits, focusing on those of period $T$ and $2 T$ (where $T=2 \pi / \Omega$ is the period of the driving) since these correspond to the dominant motions. Also quasiperiodic and chaotic orbits bifurcating from these will play an important role in our analysis.

Each periodic orbit corresponds to one or more fixed points in stroboscopic phase space and its linear stability properties are reflected by four eigenvalues. When all eigenvalues lie inside the unit circle the orbit is stable, which means that it is an attractor in phase space. When one of the four eigenvalues lies outside the unit circle the orbit is called semi-stable; for all practical purposes, of course, such an orbit is not stable. An orbit is totally unstable when two of its eigenvalues lie outside the unit circle.

From Fig. 1 it is clear that the system (but not necessarily its motion) is unchanged by the operations of reflection, exchange of pendulums, and time translation by one driving period. Alternatively stated, the equations of motion (1.1) are equivariant ${ }^{4}$ with respect to the symmetry transformations

$$
\begin{aligned}
& \mathbf{R}:\left(\vartheta_{1}, \dot{\vartheta}_{1}, \vartheta_{2}, \dot{\vartheta}_{2}, t\right) \rightarrow\left(-\vartheta_{1},-\dot{\vartheta}_{1},-\vartheta_{2},-\dot{\vartheta}_{2}, t\right), \\
& \mathbf{E}:\left(\vartheta_{1}, \dot{\vartheta}_{1}, \vartheta_{2}, \dot{\vartheta}_{2}, t\right) \rightarrow\left(\vartheta_{2}, \dot{\vartheta}_{2}, \vartheta_{1}, \dot{\vartheta}_{1}, t\right), \\
& \mathbf{T}:\left(\vartheta_{1}, \dot{\vartheta}_{1}, \vartheta_{2}, \dot{\vartheta}_{2}, t\right) \rightarrow\left(\vartheta_{1}, \dot{\vartheta}_{1}, \vartheta_{2}, \dot{\vartheta}_{2}, t+2 \pi / \Omega\right) .
\end{aligned}
$$

These are not necessarily the only symmetries of the system (we have ignored the rotational symmetry) but they are the ones relevant in the present context. It should be noted that the three transformations $(1.6(\mathrm{a})-(\mathrm{c}))$ are their own inverse, i.e., applying a symmetry transformation twice is the same as doing nothing at all. Note that we

\footnotetext{
${ }^{4}$ The equation $\dot{x}=g(x, t)$ is said to be equivariant with respect to the operation $\eta$ if $\eta \dot{x}=g(\eta x, t)$.
} 
restrict ourselves here to motions which repeat themselves every $2 T$ seconds. For these motions $t=2 T$ can be identified with $t=0$ and is $\mathbf{T}$ its own inverse.) Every combination of these symmetries is also a symmetry of the system; thus, the operations $R, E$ and $T$ generate the symmetry group $\boldsymbol{Z}_{2}(\mathbf{R}) \times \boldsymbol{Z}_{2}(\mathbf{E}) \times \boldsymbol{Z}_{2}(\mathbf{T})$. Here $\boldsymbol{Z}_{2}$ represents the so-called cyclic group of order 2 , which is to say that it contains two elements, namely, the identity transformation 1 and a non-trivial element. Now, the oscillations we are interested in possess all, some or none of these symmetries. Motions with a lesser degree of symmetry are created by means of equal period bifurcations, also known as symmetry breaking bifurcations. That is, when a motion with symmetry group structure $Z_{2} \times Z_{2}$ undergoes a symmetry breaking bifurcation the resulting motion will have a group structure $Z_{2}$. Analogously, the symmetry broken daughter of a motion with group structure $Z_{2}$ will be invariant under the identity transformation only. We will come back to the symmetry approach in detail in a forthcoming paper [5], but in the present paper we already use some of the results derived there (for instance, that the presence of certain symmetries in the oscillations can prohibit period doubling or Hopf bifurcations).

It is also worthwhile to note that the system does not possess the symmetry of time-reversal. That is, the equations of motion (1.1) with $\gamma>0$ are not equivariant with respect to the transformation $\left(\vartheta_{1}, \dot{\vartheta}_{1}, \vartheta_{2}, \dot{\vartheta}_{2}, t\right) \rightarrow\left(\vartheta_{1},-\dot{\vartheta}_{1}, \vartheta_{2},-\dot{\vartheta}_{2},-t\right)$; this symmetry is only present in a Hamiltonian context, for which $\gamma=0$. We will witness the consequences of this throughout the paper, and especially in Section 3.3, where we discuss the mixed motions.

The possible motions in this system can be divided into four categories, depicted in Fig. 2. First of all, we have the motion in which both pendulums move only in the vertical direction (following the bar), with $\vartheta_{1}(t)=\vartheta_{2}(t)=0$ for all time. This is called the downward equilibrium motion, or 0-motion. This trivial motion is indeed a special one, since it is the only motion which possesses all the symmetries present in the equations of motion.

The second motion type is the one for which both pendulums move in phase with each other, i.e., $\vartheta_{1}(t)=\vartheta_{2}(t)$; we denote them as 1 -motions. It is evident that all 1 -motions possess the exchange symmetry $(\mathbf{E})$, i.e., exchanging the pendulums does not change the motion (see also Fig. 2). In phase space the orbit of such a motion lies in the (invariant) plane for which $\phi_{2}=0, \dot{\phi}_{2}=0$, known as the 1 -plane. Eigenvalues associated with directions parallel to the 1-plane will be called 1-eigenvalues.

For the third type of motion both pendulums move in exact counterphase with each other, i.e., $\vartheta_{1}(t)=-\vartheta_{2}(t)$. These motions are called 2-motions and are symmetrical with respect to the combined operation of reflection and exchange (RE). In phase space the orbit of this motion lies in the (invariant) 2-plane, defined by $\phi_{1}=0, \dot{\phi}_{1}=0$. Eigenvalues associated with directions parallel to the 2-plane will be called 2-eigenvalues.

Finally, we have the M-motions, or mixed motions. All motions which cannot be characterized as either a 0-, 1- or 2-motion fall into this category. In general, the orbit in phase space of an M-motion is not restricted to a two-dimensional plane but uses 


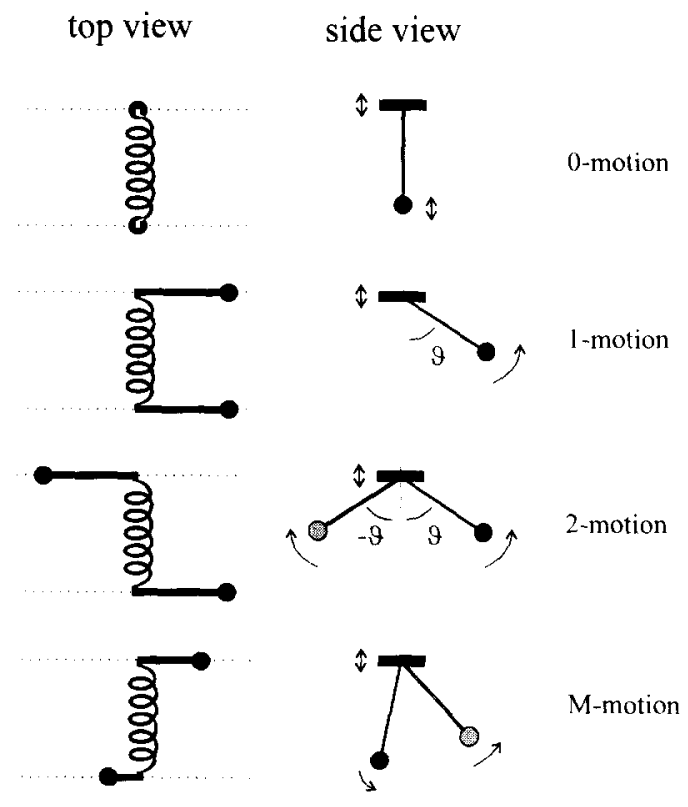

Fig. 2. The four types of motions.

all four dimensions. Motions which belong to this class can, but need not, possess any of the symmetries of the system.

We shall calculate the regions of stability in the $(A, \Omega)$-plane for the most important motions from these four categories. Special attention will be paid to the bifurcations at the borders of the stability regions. We will use the same label-convention for the bifurcation curves as in Ref. [1]. That is, a period doubling bifurcation curve (where an eigenvalue is at -1$)$ will be labeled with bold lowercase characters $(\mathbf{a}, \mathbf{b}, \mathbf{c}, \ldots)$ whereas equal period (symmetry breaking) and saddle-node bifurcation curves (an eigenvalue at +1 ) will be denoted by bold uppercase characters $(\mathbf{A}, \mathbf{B}, \mathbf{C}, \ldots)$. Hopf bifurcations form an exception to this convention and will be denoted by the character $\mathbf{H}$. It is worth noting that lines labeled with the same character, be it lowercase or uppercase, have all unfolded from the same $(K=0, \gamma=0)$-line; to distinguish the various lines we will use roman numbers as indices.

\subsection{General effects of dissipation}

The viscous damping has its effect on the position of the fixed points in phase space, as well as on their stability (governed by their eigenvalues). That is, the stability regions will be (slightly) different from their Hamiltonian counterparts. One of the most prominant changes, for each type of motion, is the appearance of a threshold in the $(A, \Omega)$-plane below which the motion cannot be excited. This threshold phenomenon can best be understood in terms of a work balance between driving and dissipation. 
It is obvious that, in order for a periodic motion to exist, the energy pumped into the system by the driving must compensate the loss of energy due to damping. This implies that the driving amplitude $A$ will have to exceed a certain threshold value, which will generally depend on $\Omega$. When we rescale time, such that $t \rightarrow \tau=\Omega t / 2 \pi$ (which means that the unit of time is now 1), our equations of motion become dimensionless

$$
\begin{aligned}
& \ddot{\vartheta}_{1}+f_{\Omega}(\tau) \sin \vartheta_{1}+\gamma_{\Omega} \dot{\vartheta}_{1}+K_{\Omega}\left(\vartheta_{1}-\vartheta_{2}\right)=0, \\
& \ddot{\vartheta}_{2}+f_{\Omega}(\tau) \sin \vartheta_{2}+\gamma_{\Omega} \dot{\vartheta}_{2}-K_{\Omega}\left(\vartheta_{1}-\vartheta_{2}\right)=0 .
\end{aligned}
$$

Here the dot represents differentiation with respect to $\tau$. The (dimensionless) expressions for the drive-function, the coupling parameter and the damping coefficient are

$$
\begin{aligned}
& f_{\Omega}(\tau)=\frac{4 \pi^{2}}{l}\left(\frac{g}{\Omega^{2}}+A \cos 2 \pi \tau\right), \\
& K_{\Omega}=\frac{4 \pi^{2}}{\Omega^{2}} K, \\
& \gamma_{\Omega}=\frac{2 \pi}{\Omega} \gamma .
\end{aligned}
$$

Apparently, the damping in natural units is inversely proportional to $\Omega$. We might therefore expect the threshold to depend on $\Omega$ in roughly the same way. This is a good first estimate, but the actual threshold curve is seen to go to infinity even faster than $1 / \Omega$. A more refined estimate, suggesting that the threshold goes to infinity roughly as $1 / \Omega^{2}$, is given in Appendix A.

Also in the (linearized) stroboscopic map the relevant damping coefficient is $\gamma_{\Omega}$ rather than just $\gamma$. This can for instance be seen in the expression for the product of the four eigenvalues of an orbit with period $n T$ (i.e., the Jacobian of the $n$ th-iterated map), which reads

$$
\lambda_{1} \lambda_{2} \lambda_{3} \lambda_{4}=\left(e^{-2 \gamma_{s}}\right)^{n}=e^{-4 \pi m_{i} / \Omega} .
$$

Also the sum of the eigenvalues (i.e., the trace of the $n$ th-iterated map) is a function of $\gamma_{\Omega}$. Eq. (1.9) shows that the eigenvalues no longer travel on the unit circle (as in the Hamiltonian system) but on a circle with radius $e^{-n_{i s} / 2}$, depending on $\Omega$, from now on called the reduced circle. Bifurcations, of course, still correspond with an eigenvalue breaking through the unit circle.

What then happens to the actual orbits themselves? Generally, the maximum deflection of both pendulums will be less than in the Hamiltonian case. Furthermore, motions that were 'in tune' with the driving for $\gamma=0$ (with the pendulums reaching their amplitude the moment the bar of suspension goes through its lowest point) will no longer have that property for $\gamma>0$. This is demonstrated in Fig. 3, which depicts the trajectory of a 1-motion in both the Hamiltonian and in the dissipative system. In phase space this means that the fixed points move away from the axes. This is directly 


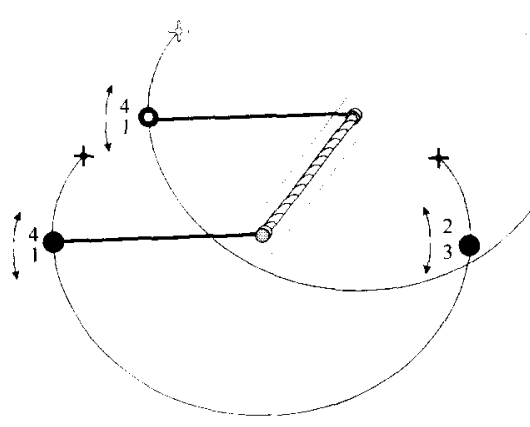

(a)

$$
\gamma=0
$$
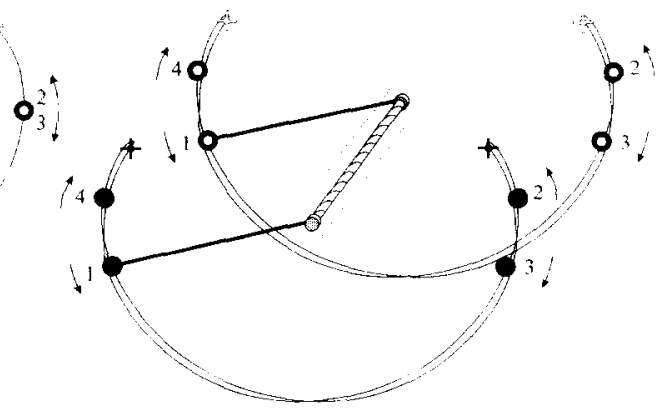

(b)

$$
\gamma=0.1
$$

Fig. 3. The real-space trajectory of a 1-motion in (a) the Hamiltonian system and (b) the dissipative system. In this figure (and those to follow) the numbers 1 and 3 denote the position of the pendulums the moment the bar of suspension passes its middle position in the upward direction; numbers 2 and 4 give the positions when the bar of suspension passes its middle position going downward. The crosses indicate the moments of stroboscopic sampling (when the suspension bar goes through its lowest position).

related to the fact already mentioned in the previous subsection, that the timereversal symmetry is no longer present in the dissipative system.

These, in a nutshell, are the general features of dissipation. The specific effects may of course vary from motion to motion, and will be discussed in the next sections. In Section 2 we treat the 0 -motion, the 1-motions and the 2-motions; Section 3 deals with the mixed motions. Section 4 gives the results of a numerical experiment we performed to establish which motion types prevail in a practical situation, i.e., for initial conditions close to the 0-motion. Finally, in Section 5, we summarize the main results and draw a comparison with related mode competition experiments, e.g. with that of the compound pendulum by Skeldon et al. [3,4].

\section{The low-dimensional motions}

\subsection{The 0-motion}

The stability of the trivial motion, or 0 -motion, is determined by the linearized equations of motion

$$
\begin{aligned}
& \ddot{\phi}_{1}+f(t) \phi_{1}+\gamma \dot{\phi}_{1}=0, \\
& \ddot{\phi}_{2}+(f(t)+2 K) \phi_{2}+\gamma \dot{\phi}_{2}=0 .
\end{aligned}
$$

These are two damped Mathieu equations. The trivial solution of these equations is stable throughout the $(A, \Omega)$-plane except within a series of tongue-shaped regions of parametric resonance. The white regions in Fig. 4a depict the most important two of 


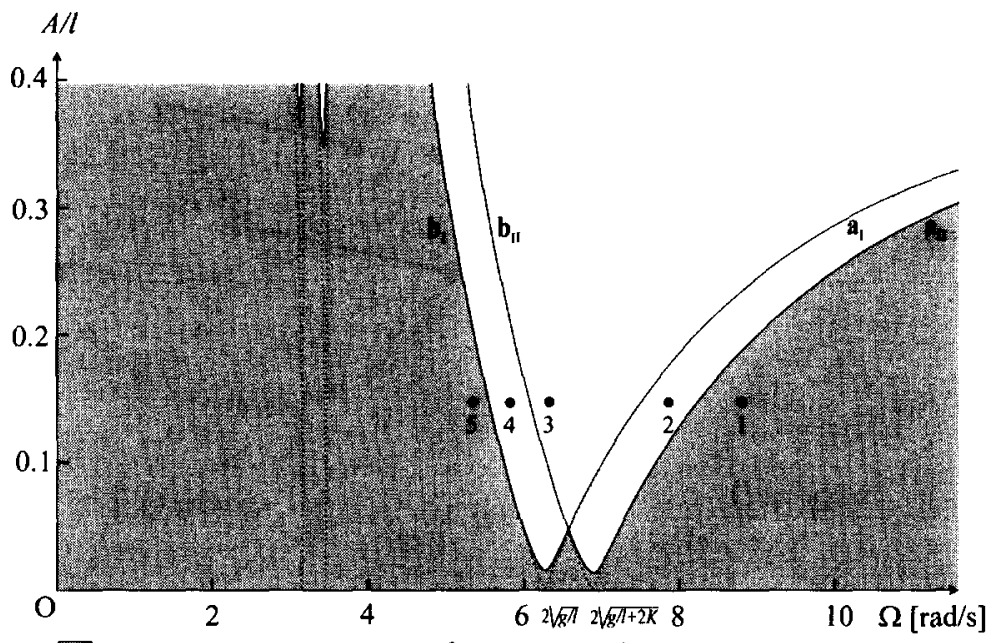

(a) stable 0 -motion (for $K=1 \mathrm{~s}^{-2}$ and $\gamma=0.1 \mathrm{~s}^{-1}$ )
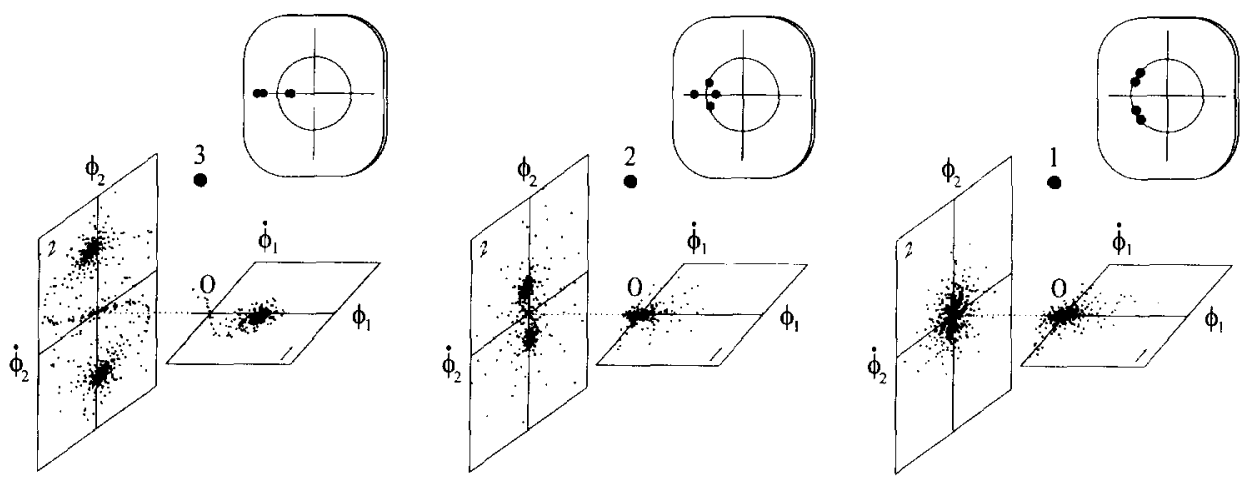

(b)
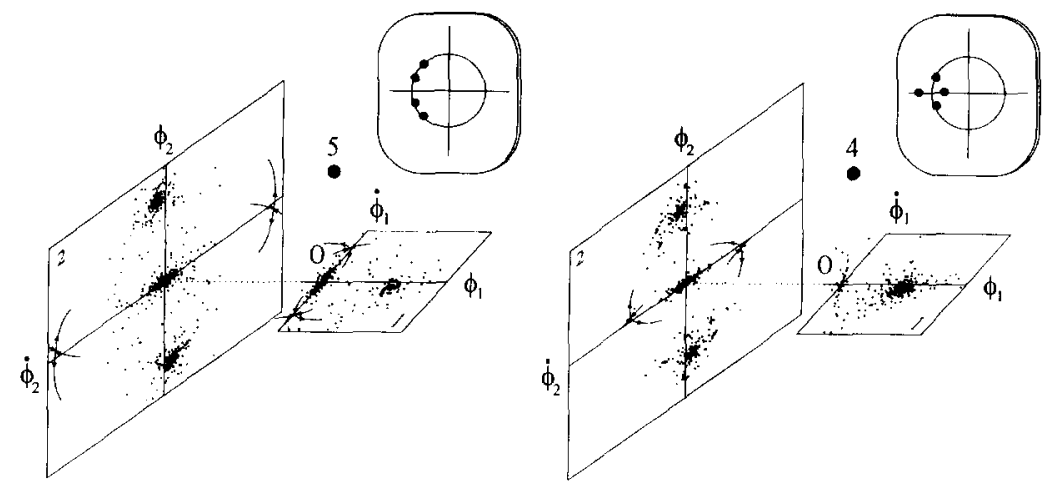

Fig. 4. (a) Stability diagram of the 0-motion, for $K=1 \mathrm{~s}^{-2}$ and $\gamma=0.1 \mathrm{~s}^{-1}$; (b) Eigenvalues and (stroboscopic) phase space portraits at the five points marked in (a). The reduced eigenvalue-circle, which lies just inside the unit circle, shrinks as one goes from 1 to 5 , but the difference is too small to be discernible. The positions of the unstable $\beta$ motions (near the $\dot{\phi}$ axes in situations 4 and 5) are indicated by saddles. 
these tongues; the others, to the left of the main tongues, have been shifted by the dissipation to large values of $A / l$. Only the tips of the second pair of tongues fall within the range of the picture. In Fig. 4a, and all figures to follow, the dotted lines serve as a guide to the eye and represent the bifurcation curves for the Hamiltonian case [1].

The left tongue is related to the instability of the 0 -motion with respect to the 1 -plane. The boundaries of this tongue, for not too large values of $A / l$, are given by (see Appendix B).

$$
\frac{A}{l}=\frac{1}{2} \sqrt{\left(1-4 \frac{g / l}{\Omega^{2}}\right)^{2}+4 \frac{\gamma^{2}}{\Omega^{2}}} .
$$

Likewise, the right tongue corresponds to the instability of the 0-motion with respect to the 2-plane. Its boundaries are given by

$$
\frac{A}{l}=\frac{1}{2} \sqrt{\left(1-4 \frac{g / l+2 K}{\Omega^{2}}\right)^{2}+4 \frac{\gamma^{2}}{\Omega^{2}}} .
$$

It may be noted that the tip of the right tongue lies lower than that of the left tongue, which means that the 2-motion can be excited somewhat more easily than the 1motion. This has to do with the fact that the effective dissipation $\gamma_{\Omega}=2 \pi \gamma / \Omega$ is weaker for the right tongue than for the left one. Another interesting observation is that the intersection point of the two tongues lies at a fixed value of $\Omega$ (given by Eq. (B.12) in Appendix B), not depending on the amount of dissipation.

At the borders of these tongues non-trivial motions come into existence as bifurcation products of the 0 -motion. This can be seen in Fig. $4 \mathrm{~b}$, where the 0 -motion's eigenvalues and numerical data of the (stroboscopic) phase space at the five points marked in Fig. 4a are presented. Along line $\mathbf{a}_{1}$ one of the 1-eigenvalues breaks through the unit-circle at -1 , and a 1-motion of period $2 T$ is born by means of a period doubling bifurcation. We shall denote this 1 -motion as a $1 \alpha$-motion. At line $\mathbf{b}_{\mathbf{t}}$ the same eigenvalue goes through -1 again, in the opposite direction. Here a reverse period doubling bifurcation takes place, rendering the 0 -motion stable once more and giving birth to another 1 -motion of period $2 T$, which we shall call the $1 \beta$-motion. Analogously, along lines $\mathbf{a}_{\mathrm{II}}$ and $\mathbf{b}_{\mathrm{II}}$ one 2-eigenvalue of the 0 -motion passes through -1 . Here the 0 -motion gives birth to a $2 \alpha$ - and a $2 \beta$-motion, respectively.

\subsection{The 1-motions}

When the 0-motion loses and regains stability in the 1-direction, crossing lines $\mathbf{a}_{\mathbf{I}}$ and $\mathbf{b}_{\mathbf{I}}$ from right to left in the $(A, \Omega)$-plane, it gives birth to a $1 \alpha$ - and a $1 \beta$-motion (see Fig. 5a and Fig. 5b). The symmetry group of these motions is given by $\boldsymbol{Z}_{2}(\mathbf{R T}) \times \boldsymbol{Z}_{2}(\mathbf{E})$, containing the elements RT, E, RET and 1. For small $\gamma$ the $1 \alpha$-motion is almost in phase with the driving, the pendulums reaching their maximum deflection shortly before the suspension bar goes through its lowest point. In the $1 \beta$-motion, on 


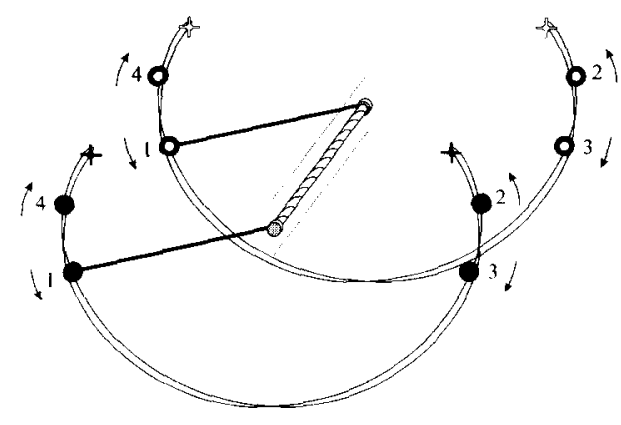

(a)

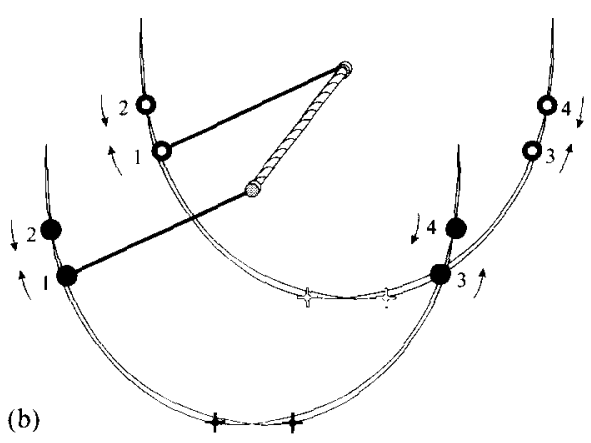

(b)

Fig. 5. (a) The $1 \alpha$-motion and (b) the $1 \beta$-motion, both for $\Omega=4.821 \mathrm{rad} / \mathrm{s}, A / l=0.138, K=1 \mathrm{~s}^{-2}$ and $\gamma=0.1 \mathrm{~s}^{-1}$. At these parameter values the $1 \alpha$-motion is stable and the $1 \beta$-motion unstable. The crosses denote the moments when the suspension bar goes through its lowest position.

the other hand, the pendulums reach their maximum speed shortly after the suspension bar reaches its lowest point. In Ref. [1] no mention was made of the $1 \beta$-motion since it never became stable. In the presence of dissipation it still does not, but the $1 \beta$-motion does play a somewhat more important role now, since it recombines with the $1 \alpha$-motion at the threshold line $\mathbf{T}_{\mathrm{I}}$. (This recombination is made possible by the fact that $1 \alpha$ and $1 \beta$ fall into the same symmetry class. We will come back to this in a forthcoming paper [5]). In Fig. 6 we have pictured the evolution of the $1 \alpha$ - and the $1 \beta$-motion as a function of $\Omega$, at a fixed value of $A / l=0.10$.

Fig. 7 depicts the stability diagram of the $1 \alpha$-motion. Going from right to left in the $(A, \Omega)$-diagram the $1 \alpha$-motion begins its life at line $\mathbf{A}_{\mathbf{l}}$, via a period doubling of the 0 -motion. Along the upper part of this line it is born semi stable, i.e., unstable with respect to the 2-direction; along the lower part it is born completely stable. At the threshold line $\mathbf{T}_{\mathrm{I}}$ the $1 \alpha$-motion ends its life when it recombines with its sister, the $1 \beta$-motion. Line $\mathbf{T}_{\mathbf{I}}$ connects to the left resonance tongue at a point very close to the tip (see also Appendix A).

Apart from the threshold effect, the changes brought about by the dissipation are not very conspicuous. Along line $\mathbf{D}_{\mathbf{I}}$ the symmetrical $1 \alpha$-motion still gives way, just as in the Hamiltonian case, to a non-symmetrical $1 \alpha$-motion by means of a symmetry breaking bifurcation [1]. The stable region of the non-symmetrical $1 \alpha$-motion has been included in Fig. 7. Subsequently, in the narrow corridor between lines $\mathbf{L}_{\mathbf{C}}$ and $\mathbf{e}_{1}$ the period doubling route to chaos can take its course.

Also the lines $\mathbf{A}_{\mathbf{U I}}$ and $\mathbf{R}_{\mathbf{I}}$ lie quite close to their Hamiltonian (dotted) counterparts. The interesting thing here is, of course, the unfolding of the former point-connection between the two parts of the stable region. As a consequence the lines $\mathbf{A}_{\mathbf{I I}}$ (which, by definition, is the birthline of the type A mixed motion) and $\mathbf{R}_{\mathbf{I}}$ (where, again by definition, the so-called Mixed Phase motion is born, see Section 3) have melted together at two places and seem indistinguishable at first sight. However, a closer inspection of both lines reveals that the bifurcation switches from supercritical so 


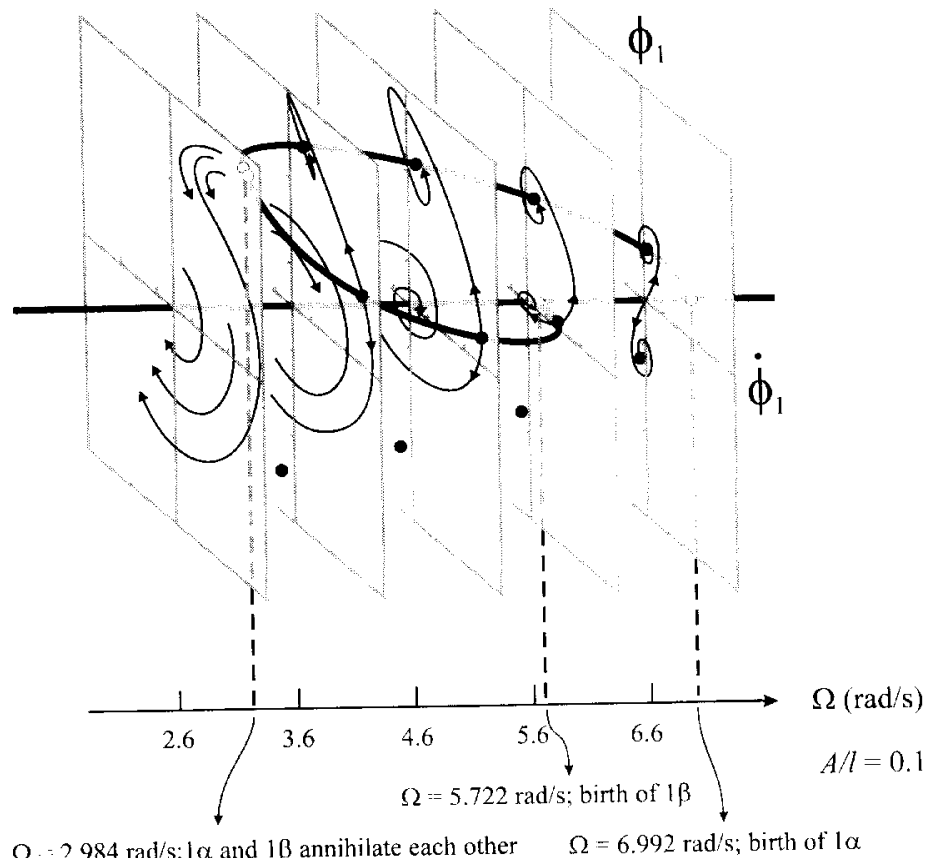

Fig. 6. A schematic representation (based on numerical results) of the fixed points of the $1 x$-and $1 \beta$-motion as a function of $\Omega$. for $A / l=0.10, K=1^{-2}$ and $:=0.1 \mathrm{~s}^{-1}$. The cross-sections are taken in the 1 -plane.

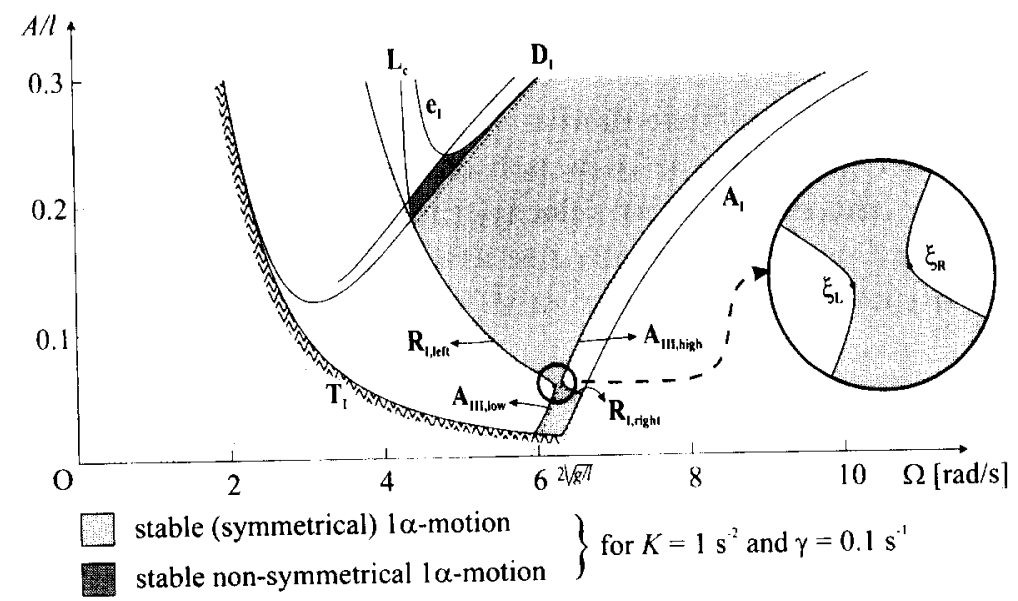

Fig. 7. Stability diagram of the $1 x$-motion, for $K=1 \mathrm{~s}^{-2}$ and $\gamma=0.1 \mathrm{~s}^{-1}$.

subcritical at the points marked $\xi_{\mathrm{L}}$ and $\xi_{\mathrm{R}}$. To preserve the analogy with the labeling of Ref. [1], we will take these points to mark the transitions from the lineparts $\mathbf{A}_{\text {III, low }}$ to $\mathbf{R}_{\mathbf{I} \text { left, }}$, and from $\mathbf{A}_{\mathbf{I I I}, \text { high }}$ to $\mathbf{R}_{\mathbf{l} \text {, right }}$ (see Fig. 7). We will come back to this in Section 3, when we discuss the mixed motions. 
For the sake of completeness we note that the $1 \alpha$-motion can also bifurcate into a mixed motion of type C (see Ref. [1]) some time after it has become unstable along line $\mathbf{D}_{\mathbf{1}}$. Because this happens only for unrealistically high values of $\Omega$ and $A / l$, we will not pursue this $\mathrm{C}$ motion.

It is perhaps good to say a few more words about the symmetry breaking bifurcation from the symmetrical $1 \alpha$-motion to the non-symmetrical $1 \alpha$-motion. Contrary to what its name suggests the latter does in fact still contain a certain degree of symmetry: its symmetry group is $Z_{2}(\mathbf{E})$. Nevertheless, it is less symmetric than its mother, which has symmetry group $\boldsymbol{Z}_{2}(\mathbf{R T}) \times \boldsymbol{Z}_{2}(\mathrm{E})$. This notation might create the impression that the bifurcation is characterized by the breaking of the combination of Reflection and Time translation (RT); there is however some ambiguity here. The symmetry group of the mother orbit contains four elements (RT, E, RET and 1) and could equally well be written as $\boldsymbol{Z}_{2}(\mathbf{R E T}) \times \boldsymbol{Z}_{2}(\mathbf{E})$; in that case one would have concluded that it was the RET symmetry that was broken. The bifurcation is in fact defined by the loss of both RT and RET. The general idea is that the only unambiguous way to characterize a symmetry breaking bifurcation is by listing all the group elements that are lost. In a $Z_{2}$ context, such as in the present paper, this list always contains $2^{n}$ elements.

\subsection{The 2-motions}

At the borders of the right instability tongue of the 0 -motion one of its 2-eigenvalues crosses the unit circle at -1 . Traversing this tongue from right to left we witness the birth of the $2 \alpha$-motion along line $\mathbf{A}_{\mathrm{II}}$ and the $2 \beta$-motion at line $\mathbf{B}_{\mathrm{II}}$; the symmetry group of these motions is equal to $\boldsymbol{Z}_{2}(\mathbf{R T}) \times \boldsymbol{Z}_{2}(\mathrm{ET})$, with elements $\mathrm{RT}, \mathbf{E T}, \mathbf{R E}$ and $\mathbf{1}$. Just as the $1 \alpha$-motion, the $2 \alpha$-motion is characterized by the fact that, for small $\gamma$, the pendulums reach their amplitude shortly before the suspension bar goes through its lowest point (see Fig. $8 \mathrm{a}$ ). For the $2 \beta$-motion, on the other hand, the pendulums reach their amplitude (and zero angular velocity) shortly after the suspension bar goes through its lowest point (see Fig. 8b). Just as the $\alpha$ and $\beta$ versions of the 1 -motion the $2 \alpha$-and $2 \beta$-motion annihilate each other in a saddle-node bifurcation at line $\mathbf{T}_{\mathbf{I I}}$ (see Fig. 9).

The stability diagram of the $2 \alpha$-motion is depicted in Fig. 9. It is born stable, as one can readily deduce from the properties of the 0 -motion along line $\mathbf{a}_{\mathbf{1 1}}$ (Fig $4 \mathrm{a}$ and Fig. 4b). Following the $2 \alpha$-motion from right to left we observe that the 1 -eigenvalues precede the 2-eigenvalues in their journey. They first make a complete tour around the reduced circle (not leaving it when they cross the negative real axis, because of their quadratic nature $[1,5]$ ), reach the positive real axis and, subsequently, one of them arrives at +1 along line $\mathbf{D}_{\mathrm{II}}$. The 2 -eigenvalues follow suit. At line $\mathbf{D}_{\mathrm{II}}$ the $2 \alpha$-motion loses its stability, becomes semi stable and gives birth to a stable mixed motion of type D. The symmetry group of this mixed motion is $Z_{2}(\mathbf{E T})$; that is, the bifurcation is characterized by the loss of the elements RT and RE. Type D is depicted in Fig. 10; its stability region is included in Fig. 9. It has a narrow stable band and soon undergoes a Hopf bifurcation, after which it does not regain its stability. 


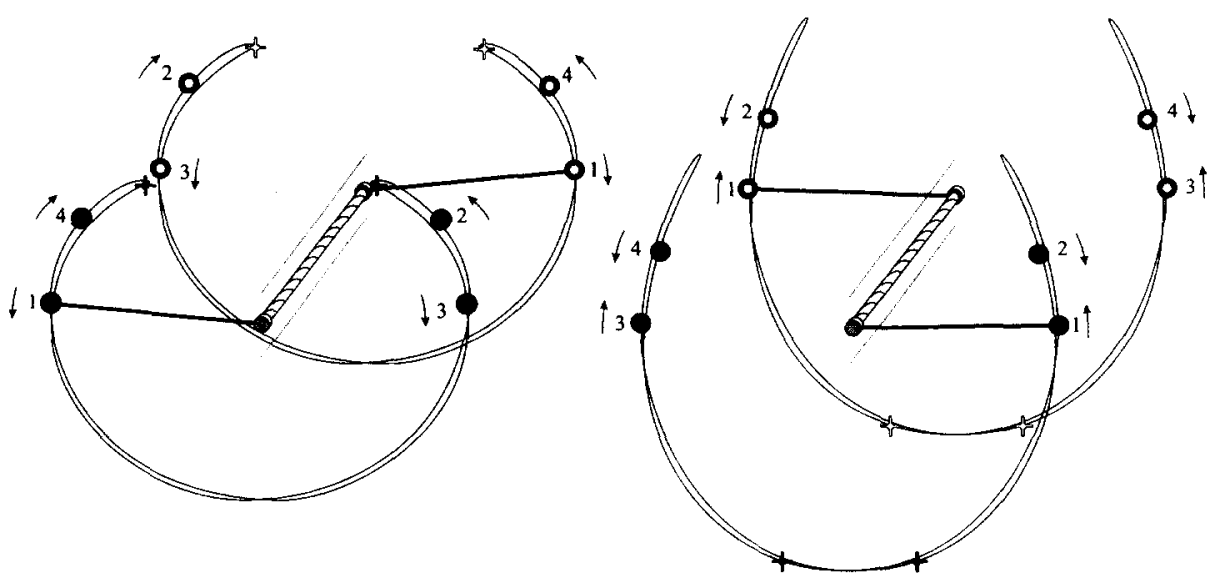

(a)

(b)

Fig. 8. (a) The $2 \alpha$-motion and (b) the $2 \beta$-motion, both for $\Omega=4.822 \mathrm{rad} / \mathrm{s}, A / l=0.162, K=1 \mathrm{~s}^{-2}$ and $\gamma=0.1 \mathrm{~s}^{-1}$. At these parameter values the $2 \alpha$ - and the $2 \beta$-motion are both semi stable.

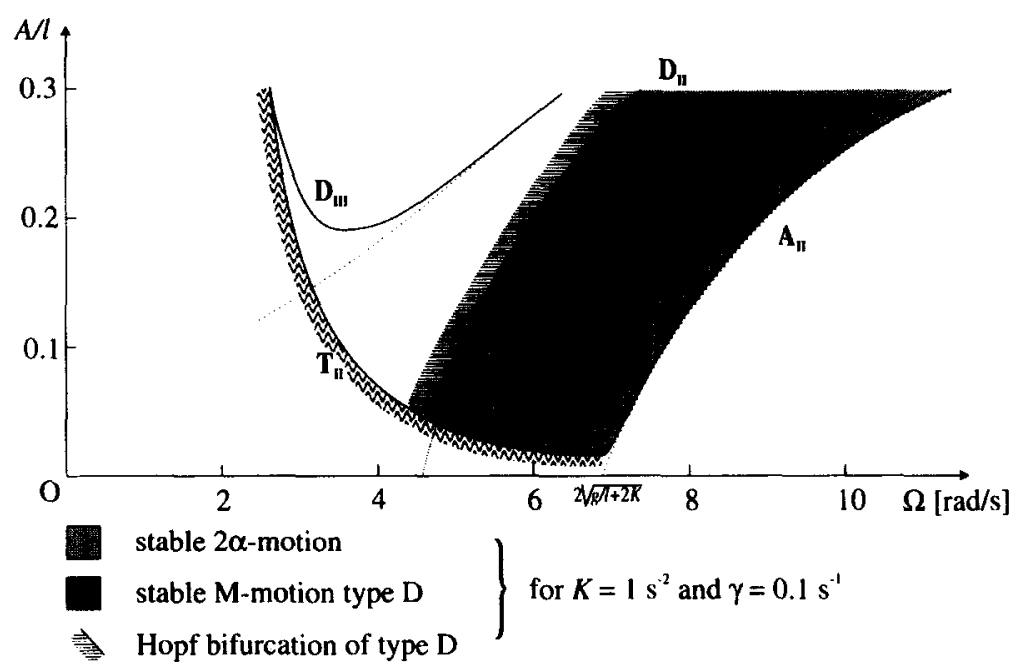

Fig. 9. Stability diagram of the $2 \alpha$-motion, for $K=1 \mathrm{~s}^{-2}$ and $\gamma=0.1 \mathrm{~s}^{-1}$.

At line $\mathbf{D}_{\text {III }}$ one of the 2-eigenvalues of the $2 \alpha$-motion goes through +1 , in the wake of the 1-eigenvalue and the symmetrical $2 \alpha$-motion gives way to a non-symmetrical version with symmetry group $Z_{2}(\mathbf{R E})$; so this bifurcation is characterized by the loss of the elements RT and ET. High in the $(A, \Omega)$-plane, where the lines $\mathbf{D}_{\mathrm{II}}$ and $\mathbf{D}_{\mathrm{III}}$ lie close to each other, this non-symmetrical motion can even have a stable region. However, because this region lies far beyond the parameter range we are interested in and since 


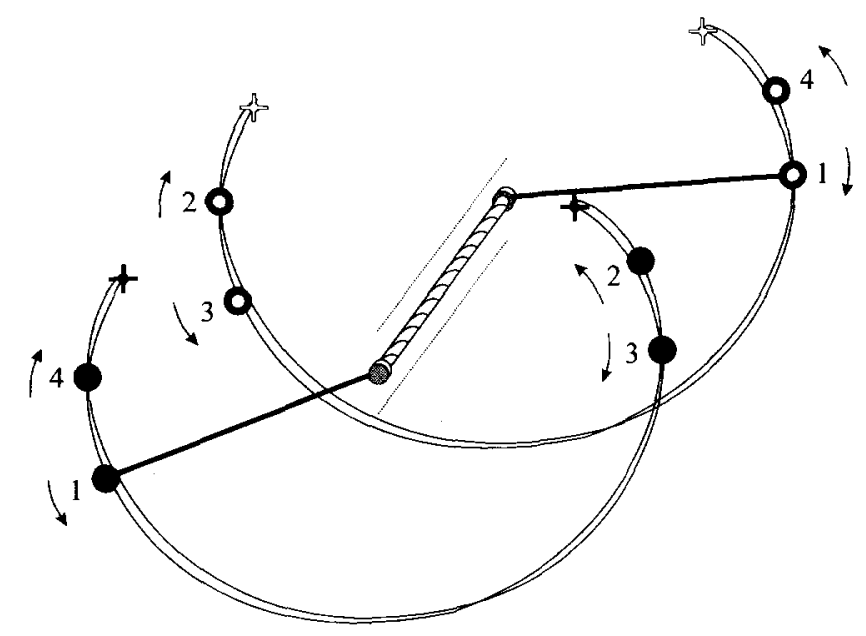

Fig. 10. Mixed motion type D, for $\Omega=5.442 \mathrm{rad} / \mathrm{s}, A / l=0.142, K=1 \mathrm{~s}^{-2}$ and $\gamma=0.1 \mathrm{~s}^{-1}$. For these parameter values type $D$ is stable.

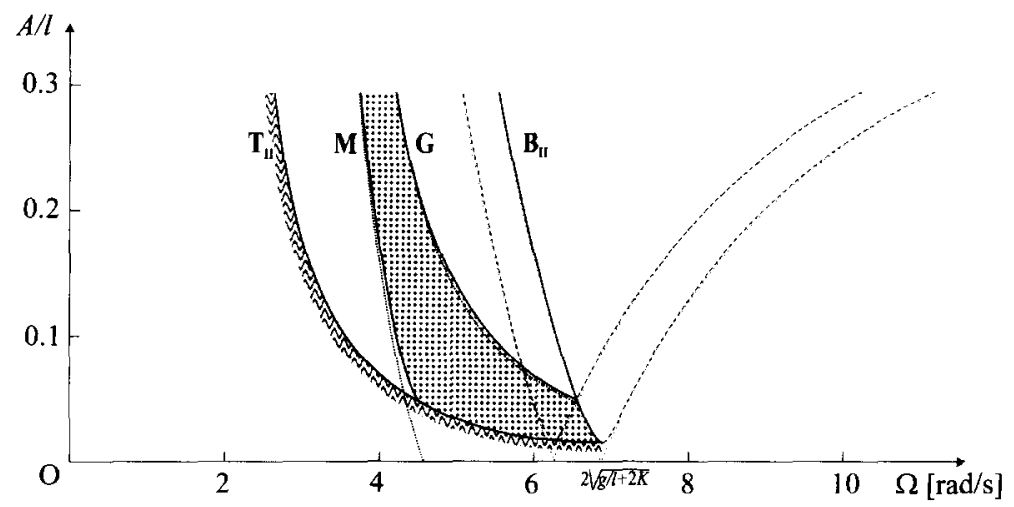

semi-stable $2 \beta$-motion (for $K=1 \mathrm{~s}^{-2}$ and $\gamma=0.1 \mathrm{~s}^{-1}$ )

Fig. 11. Stability diagram of the $2 \beta$-motion, for $K=1 \mathrm{~s}^{-2}$ and $\gamma=0.1 \mathrm{~s}^{-1}$. In the region marked with dots the $2 \beta$ motion is semi stable.

at these high values of $A / l$ the motion will certainly be outclasses by rotational and kink-like motions, we will not pursue the matter here.

Despite of the fact that the $2 \beta$-motion never becomes stable (its 2-eigenvalues reside on the positive real axis and never venture near the unit circle) it will turn out to be of crucial importance in the context of mode competition. We therefore present its stability diagram in Fig. 11. The most important line in this diagram is line G. Here, one of the 1 -eigenvalues of the $2 \beta$-motion enters the unit-circle at +1 and in the associated symmetry breaking bifurcation the mixed motion denoted as Mixed Phase is born; we will come back to this motion in Section 3. The 1-eigenvalues of $2 \beta$ travel 
around the whole reduced circle (because of the symmetry of $2 \beta$ they cannot enter the negative real axis $[1,5])$ and eventually enter the positive real axis; soon thereafter, at line $\mathbf{M}$, one of them crosses +1 , leaving the $2 \beta$-motion completely unstable.

\section{The main mixed motions}

\subsection{Introduction}

The orbits of the 0-, the 1- and the 2-motions are all described by at most two phase space coordinates. Their eigenvalues are grouped in two pairs, associated with perpendicular directions in phase space. For mixed motions this is no longer the case and a new configuration of eigenvalues becomes possible: the quadruplet $\{\lambda, \bar{\lambda}$, $\left.\lambda^{-1} e^{-n_{\eta_{2}}}, \bar{\lambda}^{-1} e^{-n_{\gamma \Omega}}\right\}$. In that case the eigenvalues leave the reduced circle not on the real axis, as before, but in the complex plane (including the imaginary axis). This happens whenever two eigenvalue pairs with opposite Krein signature meet on the reduced circle $[1,6,7]$. Two of the four eigenvalues of a quadruplet can break through the unit circle; the orbit then undergoes a Hopf bifurcation and an extra periodicity is introduced into the motion. If the position along the unit circle where the eigenvalues break through is given by $2 \pi p / q$, with $p$ and $q$ relatively prime integers (and $0<p / q \leqslant 1$, the new period introduced is $q \cdot n T$. Here $n T$ denotes the period of the original orbit, with $T$ the driving period, and the motions thus generated are said to be frequency-locked to the original motion. If, on the other hand, the position along the unit circle is given by $2 \pi \alpha$ with $\alpha$ irrational, the new periodicity is not commensurate with the original one; as a consequence the bifurcated motion will be quasiperiodic, appearing in (stroboscopic) phase space as a limit cycle.

In Ref. [1] we considered four types of mixed motions, called A, B, C and D. The most important of these, having the largest stable region in the relevant part of the $(A, \Omega)$-plane, was type $\mathrm{A}$. This mixed motion will be treated in some detail in Section 3.2. Type B is less important, but since it is a symmetry-broken byproduct of $\mathrm{A}$ it will be dealt with in the same subsection. Type $\mathrm{C}$ only occurs for unrealistically high values of the driving amplitude $A$ (see also section 2.2) and will not be treated. Type D has already been accounted for in Section 2.3 .

There are more possible mixed motions than just these four types. For instance, rotational mixed motions or kink-like mixed motions (around an equilibrium of the system in which the torsion spring is wound up) will come into play at high values of $A / l$, when enough energy is pumped into the system to sustain them. Indeed, they will eventually dominate all other kinds of motion. But because we restrict ourselves to moderate values of $A / l$ and $\Omega$ and also because we do not need them in order to explain mode competition, we will put them aside.

Another mixed motion is the Mixed Phase motion, or MP-motion. It is born from $1 \alpha$ at line $\mathbf{R}_{\mathbf{I}}$ and from $2 \beta$ at line $\mathbf{G}$; its stable region is extremely small. Mixed Phase will be seen to play a crucial role in the understanding of mode competition. Indeed, 
A and MP together form a link between $1 \alpha$ and $2 \beta$. We will devote a short subsection to this mixed motion, but leave a detailed discussion for a later paper dealing with nonlinearities in the coupling [8]. The reason for this is that, for linear coupling, the birthlines $\mathbf{R}_{\mathbf{I}}$ and $\mathbf{G}$ run very close together (especially, near the intersection point of the two resonance tongues); this can be remedied by including a third-order term in the coupling between the pendulums.

\subsection{Mixed motion types $A$ and $B$}

Along the line denoted as $\mathbf{A}_{\text {III }}$ the $1 \alpha$-motion (see Fig. 7) undergoes a symmetry breaking bifurcation and gives way to a mixed motion. The newly born motion, known as type $A$, if left with the symmetry group $Z_{2}(\mathbf{R T})$; thus, in this bifurcation the elements $E$ and RET are lost. In Fig. 12 we show the trajectory in real space of type A.

Type $\mathbf{A}$ is born stable along $\mathbf{A}_{\text {III,low }}$ and semi stable along $\mathbf{A}_{\text {III,high; }}$ its stability diagram is shown in Fig. 13, together with that of one of the A-motion's bifurcation products, the B-motion. Two major changes with respect to the Hamiltonian picture (the dotted lines) are apparent. First of all we have the appearance of a (by now familiar) threshold line, denoted as $\mathbf{T}_{\mathbf{I I I}}$. Here type $\mathrm{A}$ is being annihilated in a saddlenode bifurcation together with its counterpart bifurcated from the (unstable) $1 \beta$ motion. The second change that leaps to the eye is the unfolding of the bifurcation line where the Hamiltonian type A gained stability by means of a symmetry breaking bifurcation and in the process gave birth to the so-called ML-motion; we will come back to this motion in forthcoming papers [5,8]. In this (Hamiltonian) symmetry breaking the elements $\mathbf{t}$ and RTt were lost. The symmetry group of the dissipative

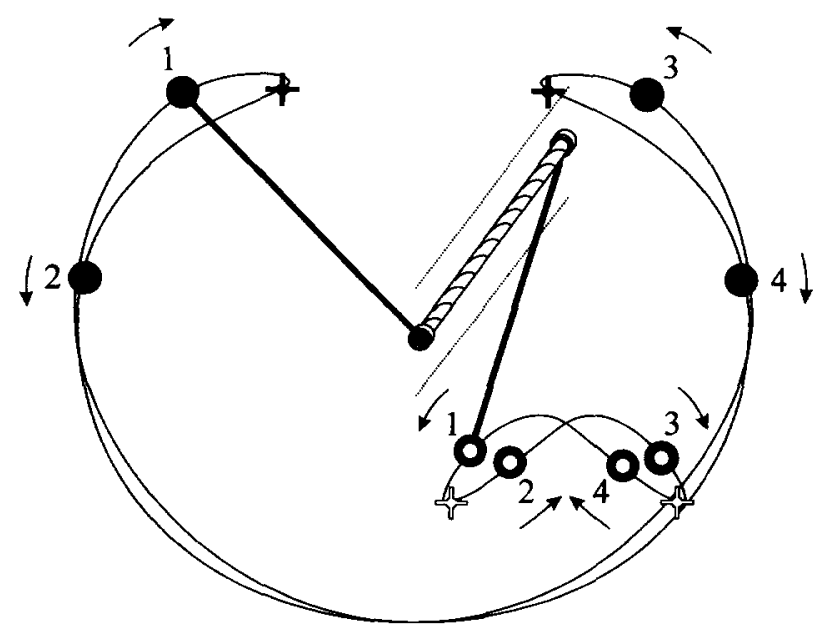

Fig. 12. Mixed motion type A, for $\Omega=3.857 \mathrm{rad} / \mathrm{s}, A / l=0.165, K=1 \mathrm{~s}^{-2}$ and $\gamma=0.1 \mathrm{~s}^{-1}$. For these parameter values type $A$ is completely unstable, with two of the four eigenvalues of the quadruplet outside the unit circle. 


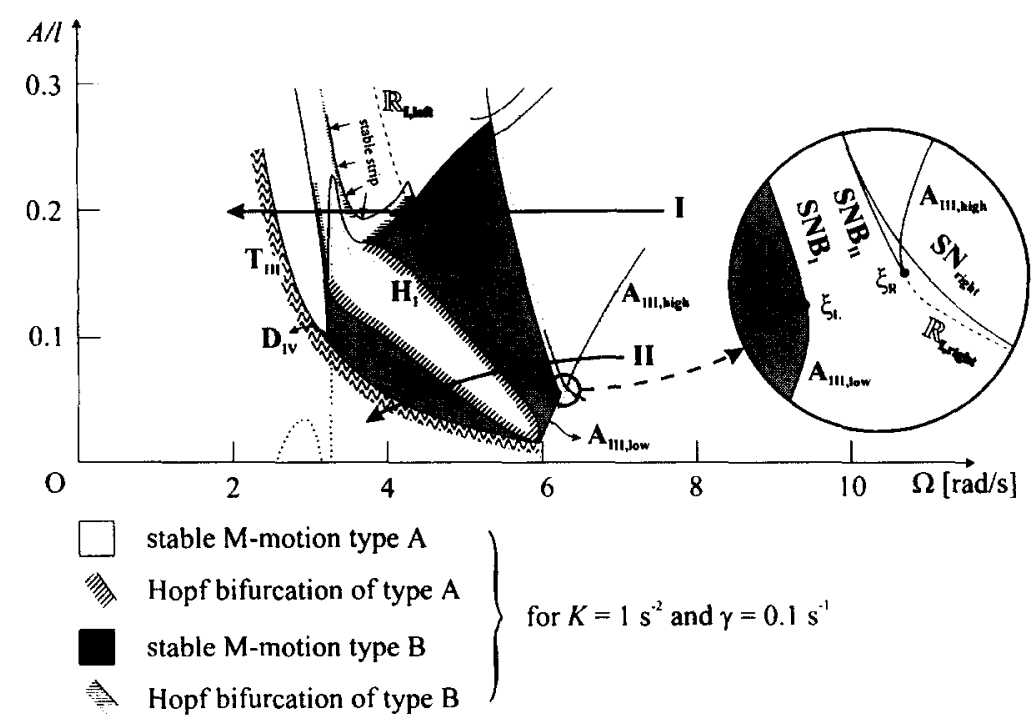

Fig. 13. Stability diagram of the mixed motions type A and B, for $K=1 \mathrm{~s}^{-2}$ and $\gamma=0.1 \mathrm{~s}^{-1}$.

system (which lacks time-reversal) does not contain these elements and they can therefore not be lost; hence the unfolding. (Stated otherwise, the former mother (A) and daughter (ML) now have the same symmetry group $\boldsymbol{Z}_{\mathbf{2}}(\mathbf{R T})$ and no symmetry breaking can exist between them). The Hamiltonian bifurcation line in question originated from the former point-connection between the stable regions of the $1 \alpha$ motion; for low values of $A$ it has been replaced by three saddle-node lines, denoted as $\mathbf{S N B}_{\mathrm{I}}, \mathbf{S N B}_{\text {II }}$ and $\mathbf{S N}_{\text {right }}$. The unfolding is schematically depicted in Fig. 14a. For higher values of $A$ the saddle-node lines $\mathbf{S N B}_{\mathrm{II}}$ and $\mathbf{S N}_{\text {right }}$ are seen to annihilate each other, resulting in the unfolding sketched in Fig. $14 \mathrm{~b}^{5}$. Lines $\mathbf{S N B}_{\mathrm{I}}$ and $\mathbf{S} \mathbf{N B}_{\mathrm{II}}$ emanate from points $\xi_{\mathrm{L}}$ and $\xi_{\mathrm{R}}$, where the bifurcation of the $1 \alpha$-motion switches from super- to subcritical (cf. Section 2.2). In the next subsection we will see that the line $\mathbf{S} \mathbf{N}_{\text {right }}$ is shared by type A and Mixed Phase.

Fig. 13 displays two other interesting bifurcation lines of the A-motion. First of all we have $\mathbf{H}_{\mathrm{l}}$, where the motion undergoes a Hopf bifurcation, as sketched in Fig. 15b. We will come back to this Hopf bifurcation shortly. Secondly, the A-motion undergoes a symmetry breaking bifurcation (in which the RT symmetry is lost) along line $\mathbf{D}_{\mathrm{IV}}$, giving rise to a mixed motion of type B. This B-motion is the first one we encounter which is void of all the symmetries considered in this paper. We will treat type B in some more detail at the end of this section.

\footnotetext{
${ }^{5}$ In Fig. 14, and in all bifurcation diagrams to follow, stable solutions are represented by solid lines, semi stable solutions by dashed lines, and totally unstable solutions by dotted lines. Bifurcations are indicated by black bullets and Hopf bifurcations have, in addition to this, a spiraled arrow.
} 


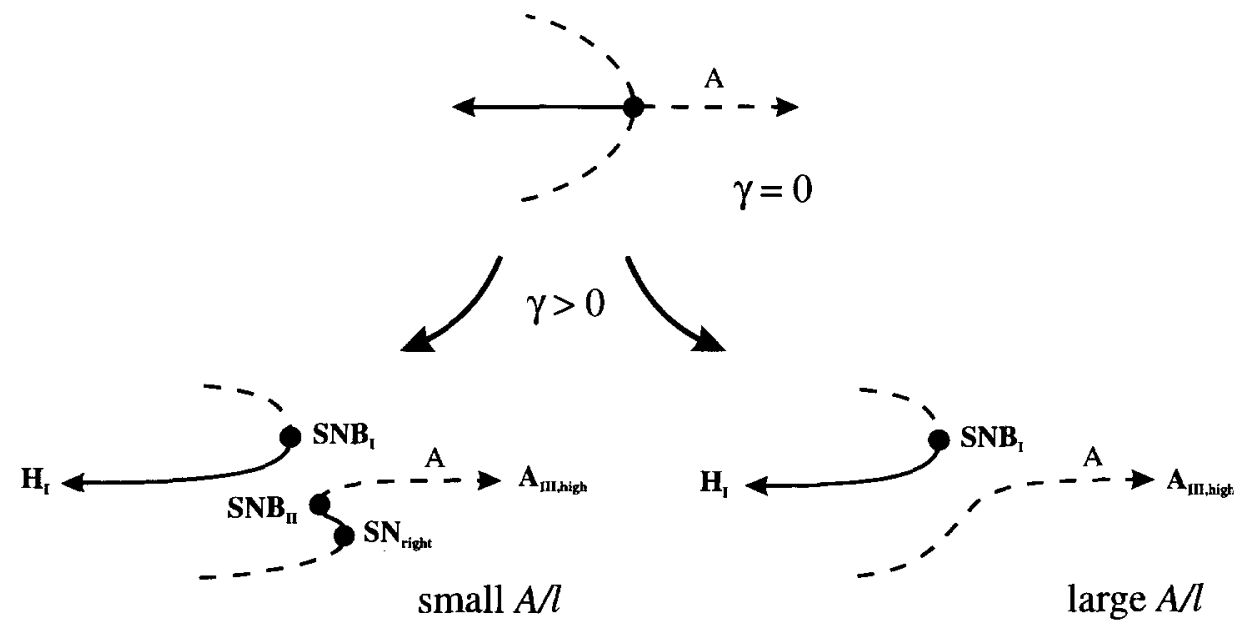

(a)

(b)

Fig. 14. Unfolding of the Hamiltonian symmetry breaking bifurcation of the type A mixed motion for (a) low values of $A / I$, (b) high values of $A / l$.

Without going into details we want to draw attention to a peculiarity in the higher regions of Fig. 13, namely the two lines curving upwards and intersecting line $\mathbf{S N} \mathbf{B}_{\mathbf{1}}$. The lower of these is a combination of two bifurcation lines, one belonging to type A (namely the upper part of line $\mathbf{D}_{\text {IV }}$ ) and the other one to the former ML-motion, which have been joined according to the unfolding depicted in Fig. 14b. Analogously, the upper curved line is a smooth combination of the period doubling line of the B-motion and a bifurcation line of one of its former daughters.

We shall now focus on the Hopf region of the A-motion, a blow-up of which is shown in Fig. 16. The eigenvalues of the A motion form a quadruplet here, and along the border of the Hopf region two of them cross the unit circle. Along the better part of the border the Hopf bifurcation is supercritical. This means that the A-motion loses its stability and simultaneously gives birth to a stable limit cycle. Along the rest of the border (the upper right-hand side) the Hopf bifurcation is subcritical, meaning that the A motion becomes unstable by swallowing an unstable limit cycle which existed outside the Hopf region. Fig. 17 depicts such an unstable limit cycle just above the Hopf region, at the point marked I in Fig. 16.

In the remainder we will leave the subcritical part of the border for what it is; we are, after all, interested in stable motions and these are generated only along the supercritical part. The stable region of the limit cycles, frequency-locked orbits en chaotic attractors is shaded in Fig. 16. The limit cycles generally undergo a quick succession of period doublings, leading to a chaotic attractor characterized by a positive Lyapunov exponent and a broad Fourier spectrum. (This is best observed in the lower right part of the Hopf region; Fig. 18 depicts a representative limit cycle before and 
(a)

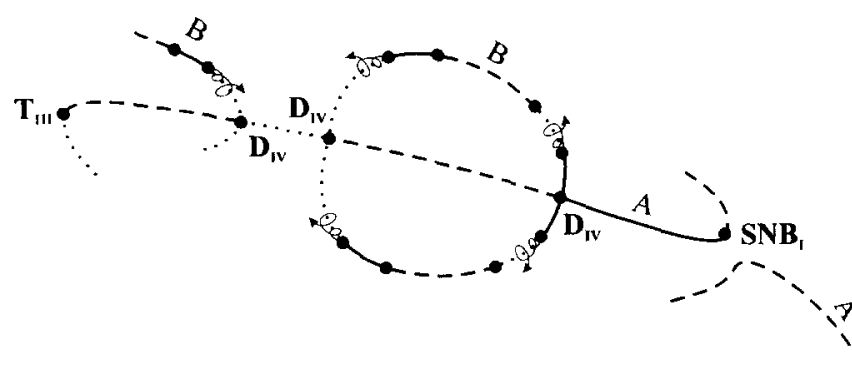

(b)

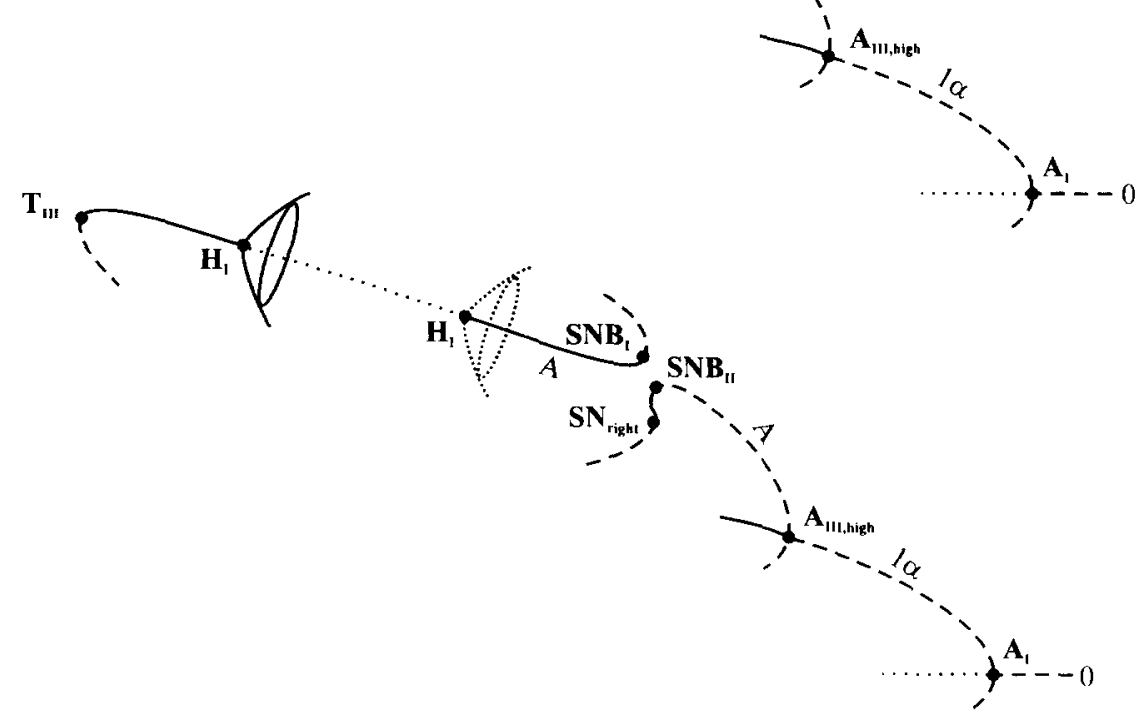

Fig. 15. (a) The bifurcational structure of the A-motion along the arrow marked I in Fig. 13. For clarity, the width of the stable regions of the B-motion has been highly exaggerated. (b) The bifurcational structure of the A-motion along the arrow marked II in Fig. 13. The stable and unstable limitcycles originating at the Hopf line $\mathbf{H}_{\mathbf{I}}$ have been indicated

after a period doubling.) The chaotic attractor can subsequently, in a symmetry restoring event, glue together with its twin sister originated from the A-motion with the pendulums interchanged. The resulting symmetrical attractor is quite large, occupying a considerable part of phase space. Further inside the Hopf region, where the shaded region ends, this attractor loses its stability by colliding with an unstable orbit; this is known as a crisis. After that we typically observe transient chaotic behavior, on the ghost of the attractor, eventually escaping to either the 0 -motion or another stable (often kink-like) motion.

The region of stable limit cycles is larded with tongues of frequency locking, commonly known as Arnol'd tongues, originating from the border of the Hopf region. Inside these tongues the extra periodicity introduced by the Hopf bifurcation is commensurate with the original period $2 T$, and the orbit takes the form of a discrete series of points. In fact, when the phase of the eigenvalue quadruplet of the A-motion is equal to $p / q$ at the moment of the Hopf bifurcation, with $p$ and $q$ relatively prime 


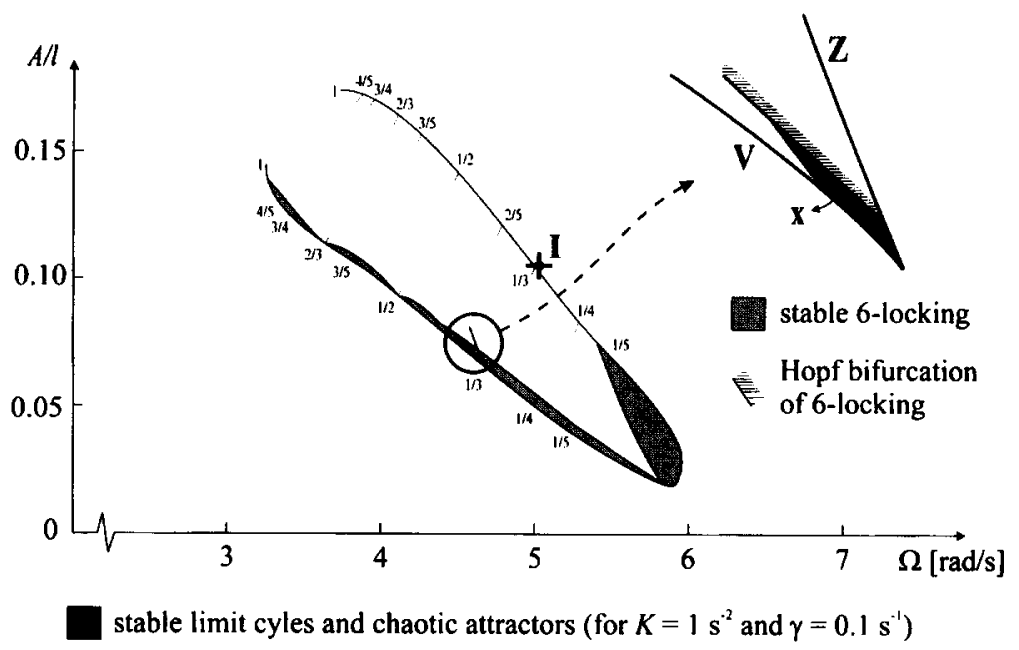

Fig. 16. Enlargement of the Hopf region of Fig. 13.

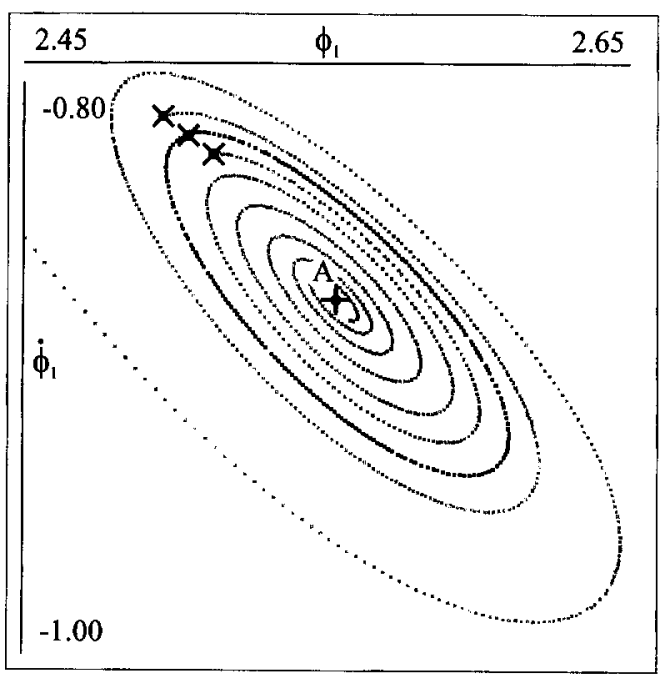

Fig. 17. Unstable limit cycle originated from the type-A mixed motion at the point marked I in Figure 16. Every 6 th iterate of the initial conditions marked by the crosses is shown.

integers, the series consists of $2 q$ points. The phase is indicated along the border of the Hopf region in Fig. 16.

In the same figure one of these Arnol'd tongue is shown in detail, namely, the $\frac{1}{3}$-tongue, which corresponds to a frequency-locked orbit consisting of 6 points. Inside the tongue we see that the orbit has a complete bifurcational life of its own. Along line $\mathbf{x}$ the 6-cycle becomes semi stable (via a period doubling to a 12-cycle). Upon entering the middle region it undergoes a Hopf bifurcation; in the narrow zone along the upper 


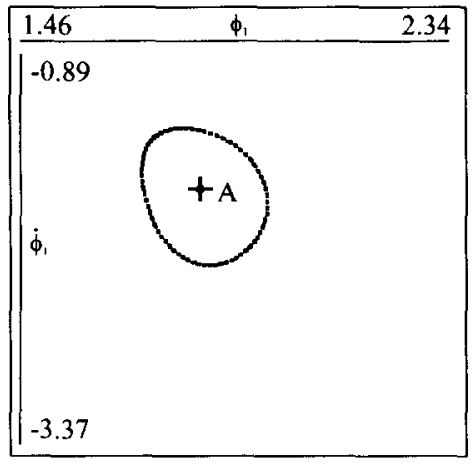

(a)

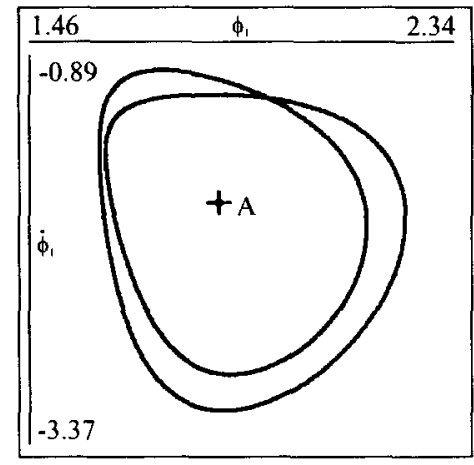

(b)

Fig. 18. Stable limit cycle originated from the type-A mixed motion, (a) before the period doubling $(\Omega=5.942 \mathrm{rad} / \mathrm{s}, A / l=0.031)(\mathrm{b})$ after the period doubling $(\Omega=5.895 \mathrm{rad} / \mathrm{s}, A / l=0.031)$.

right-hand side it is semi stable and at the borders of the tongue (lines $\mathbf{V}$ and $\mathbf{Z}$ ) it disappears altogether. Within the Hopf region of this Arnol'd tongue we find limit cycles $^{6}$ around the (unstable) 6-cycle, which in turn is centered around the unstable A-motion. An example is shown in Fig. 19a. The limit cycles rapidly grow in size and are seen to glue together, resulting in a chaotic attractor centered around the A-motion (Fig. 19b). Eventually, of course, this attractor will lose its stability just as the chaotic attractors outside the zones of frequency locking.

Looking at Fig. 16 we see yet another remarkable feature: when the phase of the eigenvalue quadruplet equals $\frac{1}{2}$ and $\frac{2}{3}$ there are no stable lockings. Recalling that the A motion is quadratic [1], the phases we are dealing with are actually those of the first iterate of the stroboscopic map: $\frac{1}{4}$ and $\frac{1}{3}$. These are strong resonances and do not give rise to frequency-locked orbits.

Finally, we briefly turn to the B motion (depicted in Fig. 20a and Fig. 20b), born from type A by means of a symmetry breaking bifurcation along line $\mathbf{D}_{\mathrm{IV}}$. Its stability diagram has been included in Fig. 13. As stated above, type B is the first $2 T$-periodic motion we encounter which does not possess any of the symmetries considered in this paper (in the bifurcation the only remaining non-trivial symmetry, RT, was lost). In the Hamiltonian case there are actually two distinct varieties of type B. The first one is born along the line where the eigenvalue pair originally (that is, for $K=0$ and $\gamma=0$ ) associated with the pendulum performing a symmetrical $2 T$ motion breaks through the unit circle. The second variety begins its existence when the eigenvalue pair originally (that is, for $K=0$ and $\gamma=0$ ) associated with the pendulum in its downward equilibrium is at +1 ; this $\mathrm{B}$-motion is closely related to the second-order tongue of

\footnotetext{
${ }^{6}$ One may also anticipate frequency-locked orbits around the 6-cycle, resulting in a self-similar structure of Arnol'd tongues within the Arnol'd tongue.
} 
(a)

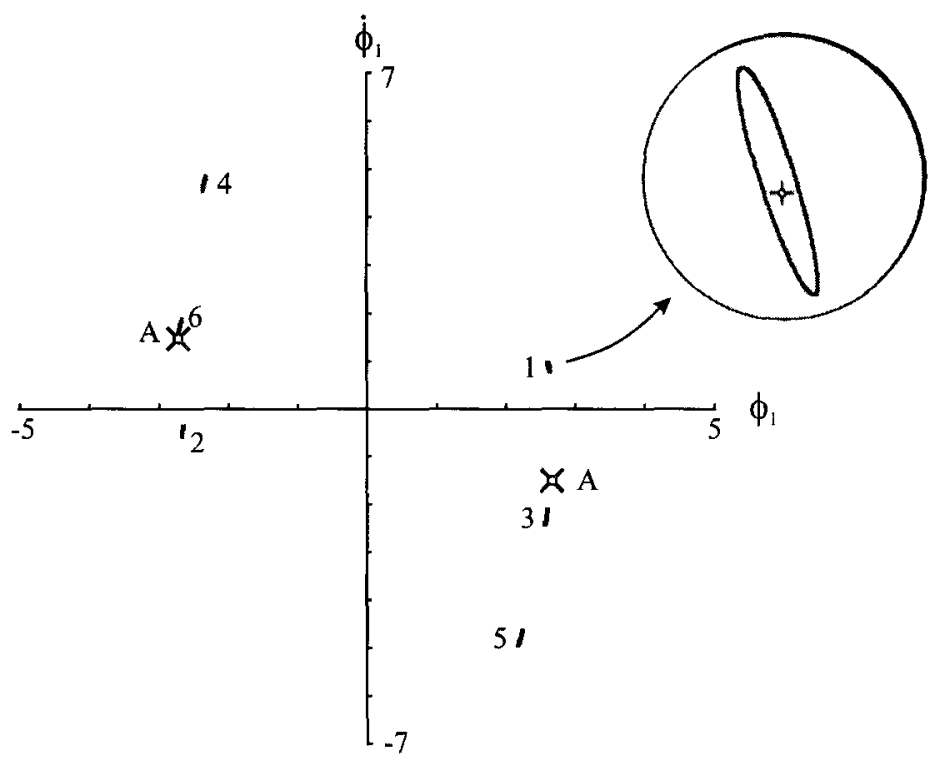

(b)

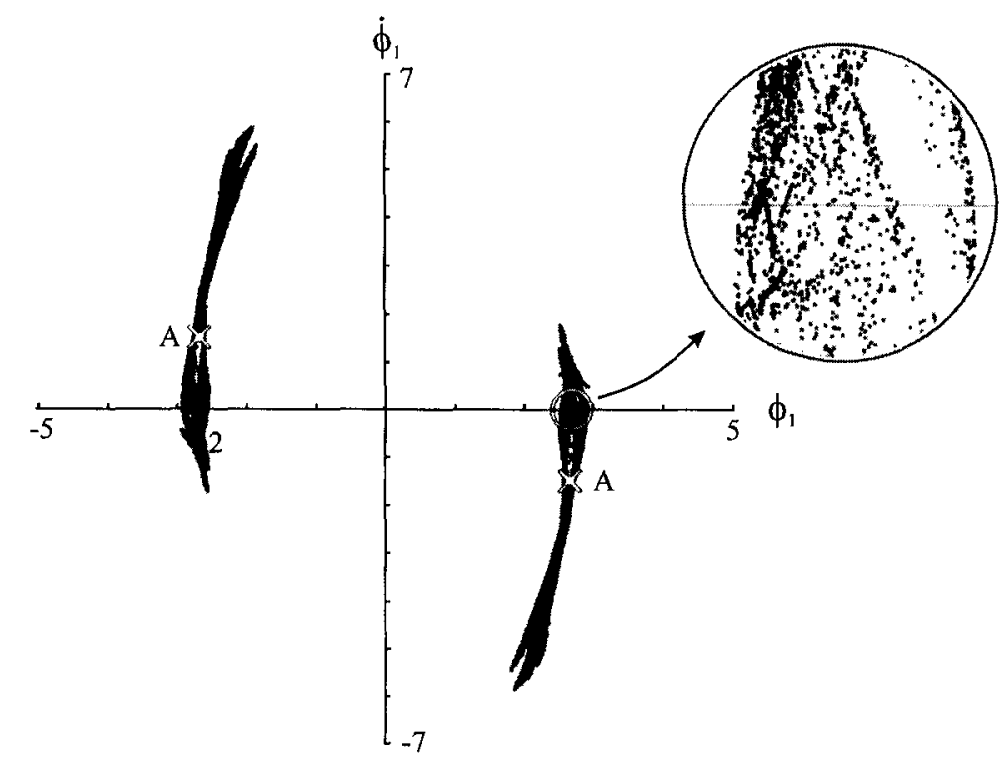

Fig. 19. (a) A Hopf bifurcated 6-cycle, i.e., 6 limit cycles, centered around the (unstable) A-motion, for $\Omega=4.617 \mathrm{rad} / \mathrm{s}, A / l=0.070, K=1 \mathrm{~s}^{-2}$ and $\gamma=0.1 \mathrm{~s}^{-1}$. (b) The next stage, $\Omega=4.617 \mathrm{rad} / \mathrm{s}, A / l=0.071$, $K=1 \mathrm{~s}^{-2}$ and $\gamma=0.1 \mathrm{~s}^{-1}$ : the limit cycles have glued together, resulting in a (symmetrical) chaotic attractor. 


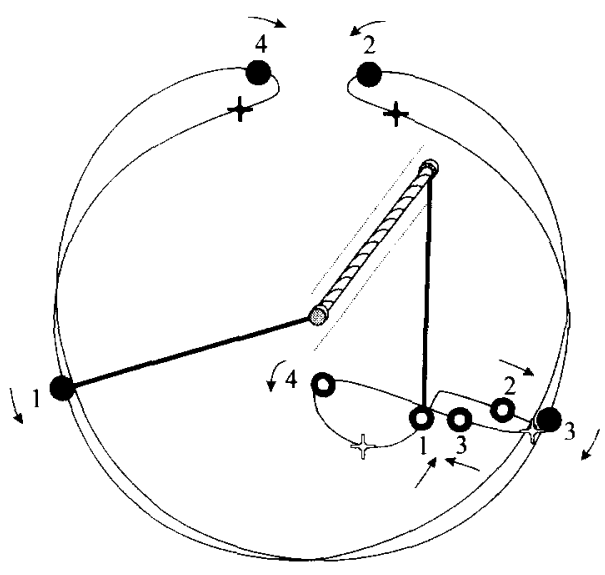

(a)

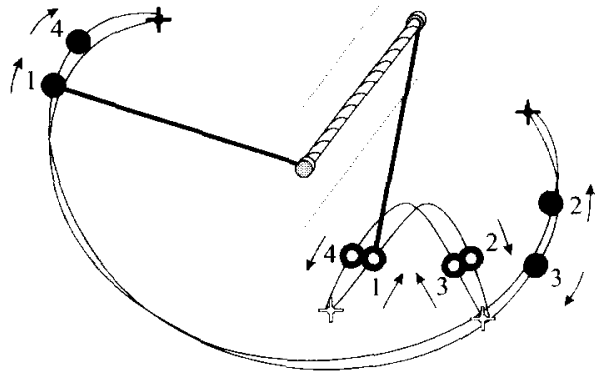

(b)

Fig. 20. Two stable Mixed motions of type B, for (a) $\Omega=3.230 \mathrm{rad} / \mathrm{s}, A / l=0.119 . K=1 \mathrm{~s}^{-2}$ and $\gamma=0.1 \mathrm{~s}^{-1} ;$ (b) $\Omega=4.926 \mathrm{rad} / \mathrm{s}, A / l=0.243, K=1 \mathrm{~s}^{-2}$ and $\gamma=0.1 \mathrm{~s}^{-1}$.

parametric resonance. The presence of dissipation has removed the distinction between the two. We thus observe three stable regions (see Fig. 13): one to the lower left side, one to the upper right side, and a very thin stable strip just above the left part of B's Hopf region. The B-motion loses its stability either by means of a Hopf bifurcation or via a period doubling. A sketch of the bifurcational structure has been included in Fig. 15a.

\subsection{Mixed Phase motion}

As mentioned in Section 3.2 there is another important mixed motion, known as the Mixed Phase (or MP) motion. It derives its name from the fact that the pendulums move neither in phase with each other (as in a 1-motion) nor in counterphase (as in a 2-motion), but have a phase difference which varies between these two extremes. The stability diagram of the MP-motion is depicted in Fig. 21. MP is born from $1 x$ along line $\mathbf{R}_{\mathbf{I}}$ and from $2 \beta$ along line $\mathbf{G}$. Indeed, in the Hamiltonian case this mixed motion forms a continuous solution-branch in the bifurcation tree from the $1 \alpha$ - to the $2 \beta$-motion. Fig. 22 depicts MP for three sets of driving parameters: one close to line $\mathbf{R}_{1 \text { l left }}$ (Fig. 22a), one close to line $\mathbf{G}$ (Fig. 22c), and one in between (Fig. 22b).

The MP-motion is born from the $1 \alpha$-motion in a symmetry breaking bifurcation. Along the segment $\mathbf{R}_{\mathrm{I}, \text { left }}$ this results in a semi stable MP-motion, and along the segment $\mathbf{R}_{\mathrm{I} \text {, right }}$ the MP-motion is born stable. In the bifurcation the elements $\mathbf{E}$ and RET are lost, which leaves MP with the symmetry group $Z_{2}(\mathbf{R T})$. (Note that this puts MP in the same symmetry class as the A-motion; indeed, the two motions can grow towards and even annihilate each other.) The connection with the $2 \beta$-motion also 


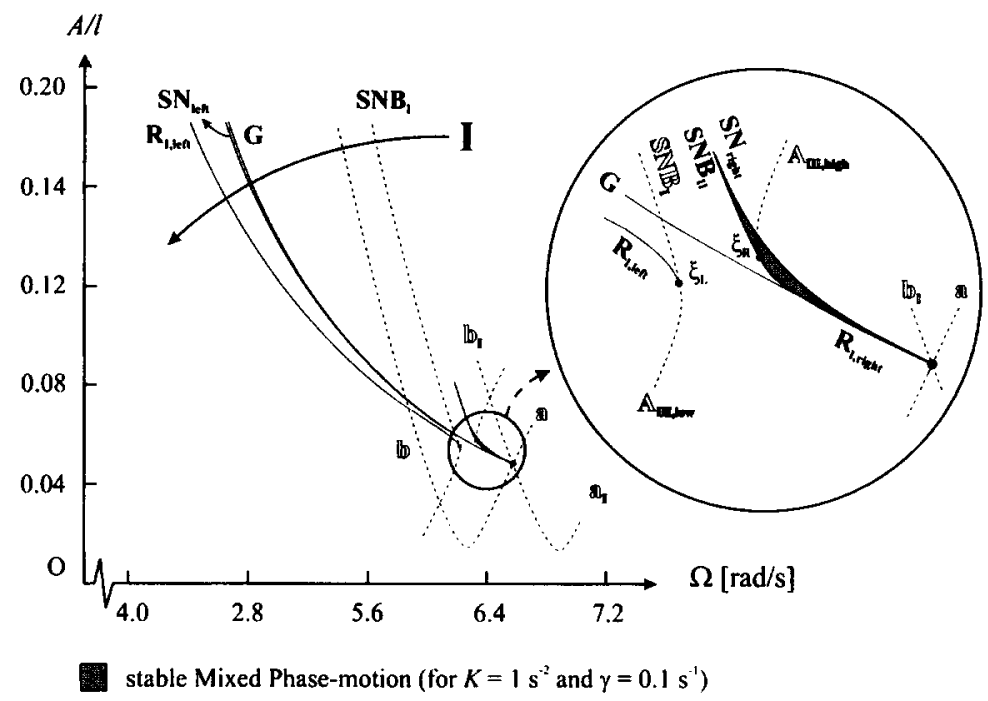

Fig. 21. The stability diagram for the MP-motion, for $K=1 \mathrm{~s}^{-2}$ and $\gamma=0.1 \mathrm{~s}^{-1}$.

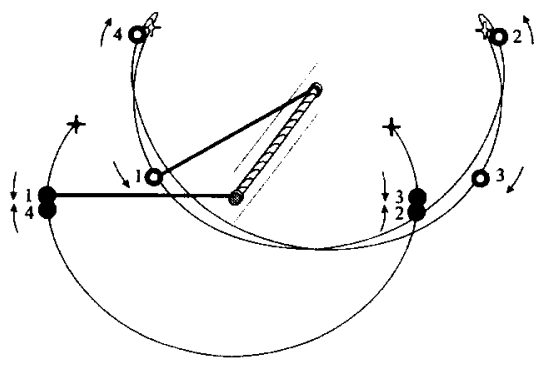

(a)

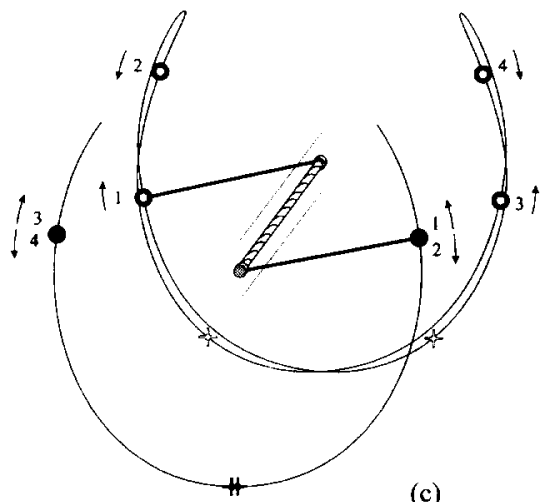

(c)

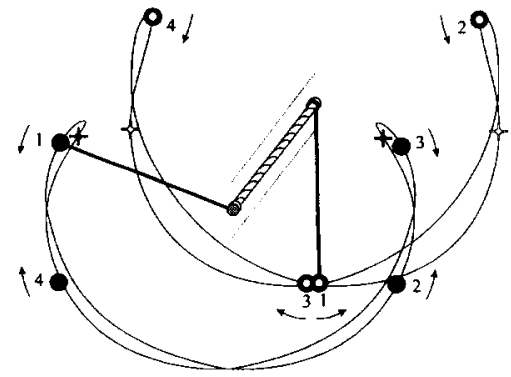

(b)

Fig. 22. (a) Semi stable MP-motion, for $\Omega=4.834 \mathrm{rad} / \mathrm{s}, A / l=0.137$ (close to line $\mathbf{R}_{1, \text { lefi }}$ ). (b) Semi stable MP-motion, for $\Omega=4.834 \mathrm{rad} / \mathrm{s}, A / l=0.155$ (midway between lines $\mathbf{R}_{\mathrm{L} \text { left }}$ and $\mathbf{G}$ ). (c) Unstable MP-motion $\Omega=4.846 \mathrm{rad} / \mathrm{s}, A / l=0.158($ close to line $\mathbf{G})$. 
happens via a symmetry breaking bifurcation. Not surprisingly, the symmetry elements lost in this bifurcation are RE and ET.

In the Hamiltonian system MP possessed a symmetry breaking bifurcation line. Just as the symmetry breaking bifurcation line of type A this line originated at the point-connection between the two stable regions of the $1 \alpha$ motion (see also the previous subsection) and involved the loss of (combinations of) time-reversal. Since these symmetries are no longer present in the dissipative system, the bifurcation has unfolded into a continuous solution-branch and a saddle-node line, denoted as $\mathbf{S} \mathbf{N}_{\text {lef }}$. The unfolding has schematically been depicted in Fig. 23. For $\gamma=0$ and also for $\gamma$ sufficiently small the MP branch is seen to contain a Hopf bifurcation, but for the present value of $\gamma\left(\gamma=0.1 \mathrm{~s}^{-1}\right)$ this Hopf bifurcation is no longer present. It has been 'swallowed' by the saddle-node bifurcation $\mathbf{S N}_{\text {left }}$.

In Fig. 24 we show the bifurcational structure along the arrow marked I in Fig. 21. From these figures the intimate relation between Mixed Phase and type A becomes clear: we can start of from the $1 \alpha$-motion at line $\mathbf{A}_{\mathrm{III} \text {, high }}$ and follow the solution branch A through a saddle-node ( $\mathbf{S} \mathbf{N}_{\text {left }}$ ) to branch MP, to finally connect with the $2 \beta$-motion at line G. Moreover, the line denoted as $\mathbf{S N}_{\text {right }}$ is shared by MP and $\mathbf{A}$. That is, close to the intersection point of the resonance tongues the stable solution branch emanating from $\mathbf{S N}_{\text {right }}$ connects with $\mathbf{R}_{1, \text { right }}$ (a birthline of the MP-motion), while higher up
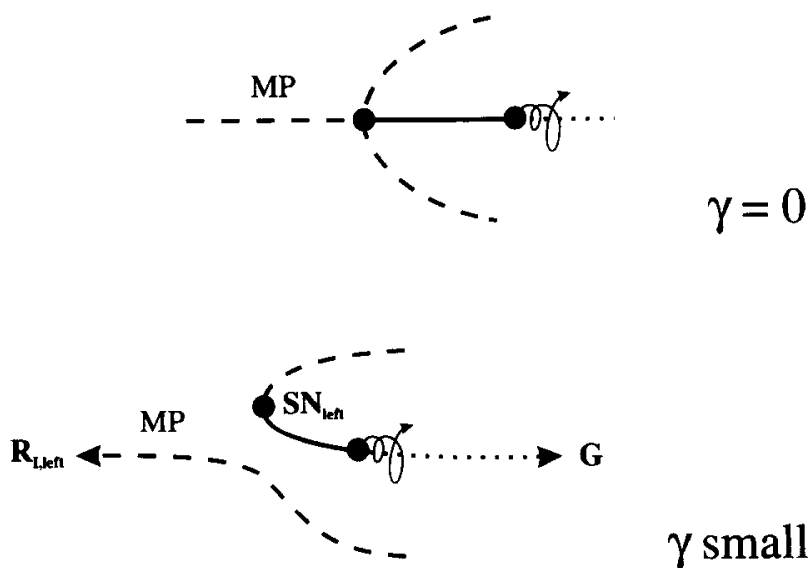

$\gamma$ small

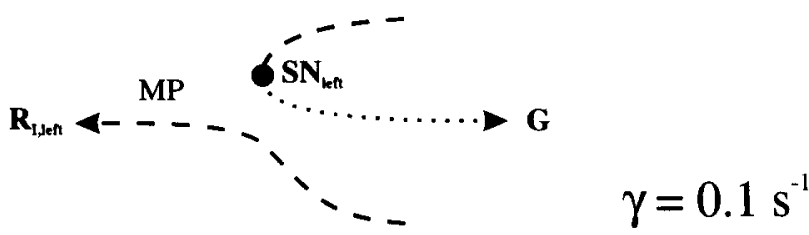

Fig. 23. Unfolding of the Hamiltonian symmetry breaking bifurcation of the MP-motion. 


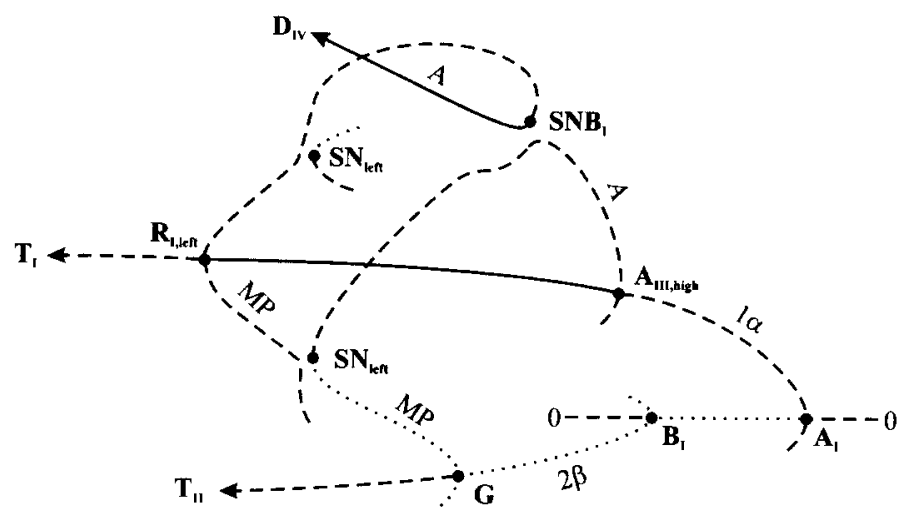

Fig. 24. Schematic representation of the bifurcational structure of the MP-motion along the arrow marked I.

in the diagram this stable branch connects with line $\mathbf{S N B}_{\mathbf{I l}}$ (associated with the A-motion). The transition from the former to the latter situation takes place at $\xi_{\mathrm{R}}$, where $\mathbf{S N B}$ II smoothly joins with $\mathbf{R}_{\mathrm{I} \text {, right }}$. All this is made possible by the fact that $A$ and MP belong to the same symmetry class, or in other words, by the dissipation.

As can be seen from Fig. 21 lines $\mathbf{R}_{\mathrm{I}}, \mathbf{S N}_{\text {left }}, \mathbf{S N}_{\text {right }}$ and $\mathbf{G}$ run extremely close together near the intersection point of the resonance tongues. Establishing the character of the interaction between $1 \alpha$ and $2 \beta$ in this region is therefore a complicated task and not very useful, since for all practical purposes the lines coincide. Indeed, analytical investigations (averaging the equations of motion and subsequently performing a center-manifold reduction) close to the intersection point of the two resonance tongues show that the problem is near-degenerate. One way to lift (part of) this degeneracy is to include extra non-linear terms in our equations of motion, for instance a third-order coupling term. A more detailed discussion is therefore postponed to a forthcoming paper dealing with non-linear coupling [8].

\section{A numerical experiment}

We have now charted the system's most prominent motions and we have seen that in many regions of the $(A, \Omega)$-plane, for given values of $K$ and $\gamma$, various of these motions are simultaneously stable. Which of them manifests itself in practice depends on the initial conditions and on how the experiment is performed. One of the most natural choices for the initial condition in phase space is to randomly pick one close to the 0-motion, and as far as the experiment is concerned there are two standard approaches. The first one is to start with parameter values outside the resonance tongues and adiabatically change $A$ and/or $\Omega$ to enter the tongues. For example, if we traverse the tongues from right to left (adiabatically lowering the value of $\Omega$ ), we would most certainly follow the continuous transition via symmetry breaking 
bifurcations from the 0 -motion through the $2 \alpha$-motion to the type $\mathrm{D}$ mixed motion. Or, if we would start under the left resonance tongue and adiabatically increase the value of $A$ we could, for instance, witness a transition from the 0 -motion to the $1 \alpha$-motion, and then to a mixed motion of type A or MP. This is in fact the kind of experiment we have done numerically up to now. Another, equally natural experiment is to pick any combination of $A$ and $\Omega$, release the pendulums from somewhere close to the downward position, turn on the drive mechanism and observe in what motion the system eventually settles down. This will obviously be the 0 -motion in parameter regions where it is stable, but what happens inside the resonance tongues? The answer is given in Fig. 25, where the outcome of this (numerical) experiment is displayed.

The thing we actually measure in this experiment is in which motion's basin of attraction in phase space the chosen initial condition lies. (Incidentally, one and the same initial condition was used in all calculations.) In some regions of the $(A, \Omega)$ plane, when there is only one basin of attraction close to the origin, this will give an unambiguous result; in other regions, however, when there are several basins (often delicately intertwined) the outcome depends sensitively on the initial condition. In particular, when two basins of attraction occupy equal volumes of phase space near the origin, our randomly picked initial condition will have equal probability to settle in any of the two attracting motions.

It does not come as a surprise that in the non-overlapping part of the right resonance tongue the $2 \alpha$-motion (dark green) dominates; it has few, if any, rivals. On the other side, in the non-overlapping part of the left tongue, the structure of Fig. 7 is clearly visible: when $1 \alpha$ (light blue) is stable, it is often the dominant motion. (One might remark that $2 \alpha$ is also stable in most of the non-overlapping part of the left resonance tongue. However, in this region the $2 \alpha$-motion already has too large an amplitude to emerge as an attractor near the origin of phase space). Despite its strong presence, the $1 \alpha$-motion concedes some ground to type A (orange) and to kink solutions (dark purple). A typical example of a kink solution is depicted in Fig. 26. The stable region of type A (see Fig. 13) can also be recognized, and at the upper boundary one even discerns the bifurcation from type A to type B (silver gray). For even higher values of the driving amplitude one encounters a brown region. Here the experiment has not yielded any conclusive outcome even after 20000 driving periods. This means either that the convergence to a periodic or quasiperiodic motion is extremely slow, or that the pendulums are performing a chaotic motion. The brown region is seen to cover a considerable area in the upper left corner of the diagram. Towards the right (still for high values of the driving amplitude $A$ ) rotational motions (light purple) take over, interlaced with orbits of high period (dark blue) and quasiperiodic motions (yellow). In this region we also sporadically find chaotic motions; the corresponding black dots are just barely discernible. Indeed, in these high regions of the $(A, \Omega)$-plane the energy pumped into the system is so large that we encounter a lot of exotic motions. They clearly play an important role for the coupled pendulums and yet the figure shows that we were justified in not treating them. We are, after all, mainly interested in the phenomenon of mode competition (involving the 


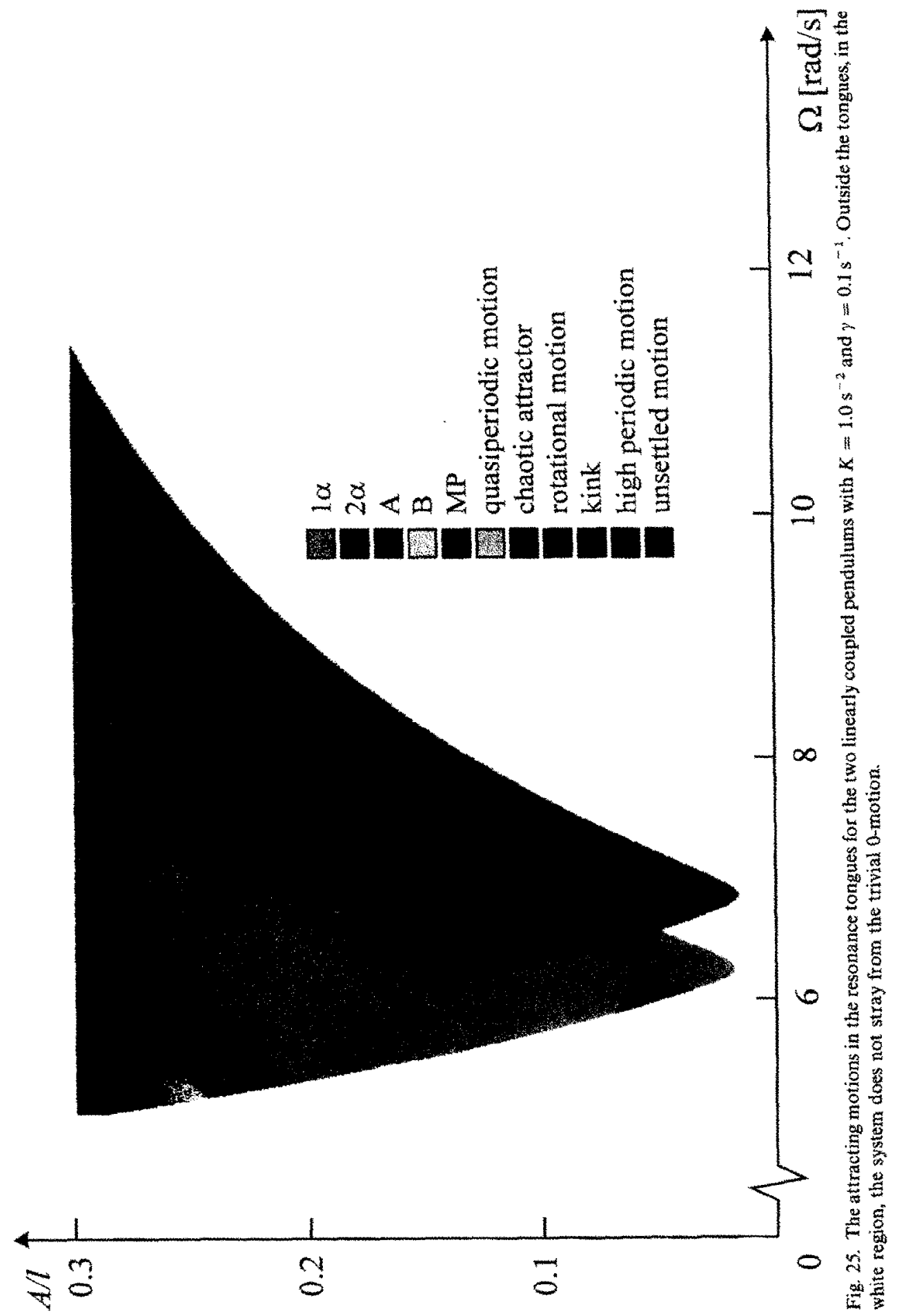




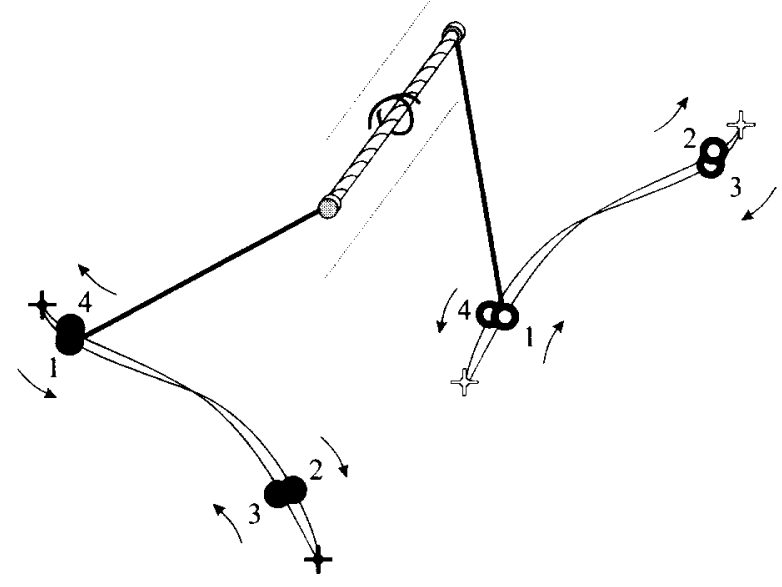

Fig. 26. Stable kink-motion (an oscillation around an equilibrium in which the torsion spring is would up once) for $\Omega=6.500 \mathrm{rad} / \mathrm{s}$ and $A / l=0.240$.

mixed motions MP and A) and this takes place at relatively low values of $A / l$, well beneath the domain of rotational and kink-like motions.

As expected, the MP-motion (red) plays a minor role in this experiment, since it is stable only within a narrow strip in the $(A, \Omega)$-plane, depicted in Fig. 21 . Under the circumstances, it is gratifying that almost the complete stable strip is visible. More precisely, in the part where MP and $2 \alpha$ are stable (but $1 \alpha$ is not) the MP-motion shows up, which means that it has a stronger hold on (the neighborhood of) the origin of phase space than $2 \alpha$. But in the part of the strip where all three are stable $(1 \alpha, 2 \alpha$ and MP) the $1 \alpha$-motion dominates.

In the region where the two resonance tongues overlap, we observe the $1 \alpha$-motion gradually taking over from the $2 \alpha$-motion. This transition does not begin immediately after stabilization of the $1 \alpha$-motion at line $\mathbf{A}_{\text {III, high }}$. This is because the $1 \alpha$-motion. when it becomes stable, has already traveled a considerable distance from the origin in phase space. Hence, its (initially small) basin of attraction has to grow a while before it enters the neighborhood of the origin. Until then the $1 \alpha$-motion does not show up in this experiment. It is interesting to note that in the higher part of transition region, where the system hesitates between $2 \alpha$ and $1 \alpha$, the pendulums often find a third way out and settle down in more complicated motion (of period higher than $2 T$ ) instead.

\section{Summary and conclusions}

In this paper, which is a continuation of Ref. [1], we have studied the dynamics of two parametrically driven, linearly coupled pendulums, subjected to viscous dissipation in the pivots. We charted the regions of stability of the prominent motions: the 
trivial motion (0-motion), the 'normal modes' (1- and 2-motions) and the mixed motions A, B, D and MP.

For the 1- and the 2-motion the most important change brought about by dissipation is the appearance of threshold curves in the $(A, \Omega)$-plane, below which the motions do not exist. These curves are seen to rise asymptotically towards the $A$-axis, indicating that for decreasing values of $\Omega$ (i.e., for growing amplitude of the pendulums) more and more energy has to be pumped into the system to overcome the dissipation.

For the mixed motions one also finds threshold curves; in addition, two other phenomena present themselves which deserve even more attention. The first of these is the quasiperiodic route to chaos. This route is made possible by the fact that the mixed motions (in contrast to the normal modes) can undergo Hopf bifurcations [5]. We used mixed motion A to demonstrate the route, because its Hopf region is so big and lies in a central part of the $(A, \Omega)$-plane. At the border of this region the A motion undergoes a Hopf bifurcation, becoming unstable. Whenever the bifurcation is supercritical stable limit cycles are created, which subsequently undergo period doubling bifurcations to end up as a chaotic attractor. Eventually, the chaotic attractor is destabilized by way of a so-called crisis, that is, it collides with the basin of attraction of, say, a 0 -motion or a kink-solution. In some cases the chaotic attractor has time to 'glue' together with another chaotic attractor, restoring some of the motion's symmetry; quite often, however, the destabilization of the attractor takes place before it has had a chance to glue, or even before it has completed its period doubling route to chaos. An interesting variation of the above sequence of events is observed when the two frequencies of a (stable) limit cycle are commensurate. This gives rise to frequency lockings in regions which are commonly known as Arnol'd tongues. The lockings turn out to have a complete bifurcational life of their own, including period doublings and Hopf bifurcations.

So much for the supercritical Hopf bifurcations. There is, however, also a considerable part of the border of the A-motion's Hopf region where the bifurcation is subcritical, involving unstable limit cycles. In this case there is no quasiperiodic route to chaos. Similar behavior is also found in the Hopf regions of the other mixed motions (B, D and MP), only on a much smaller scale in the $(A, \Omega)$-plane.

The second phenomenon, recapturing the title of this paper, is the competition between two normal modes via mixed motions. The MP-motion proves to play a crucial role here. Away from the mode interaction point (the intersection point of the two resonance tongues) MP and A together form a link between the $1 \alpha$ - and $2 \beta$-motion. This is made possible by the fact that dissipation breaks the time-reversal symmetry of the Hamiltonian system, thereby putting MP and A in the same symmetry class (see also Ref. [5]). Close to the mode interaction point the issue is less clear, due to the fact that in this region the birthlines of $M P$, lines $\mathbf{R}_{\mathbf{I}}$ and $\mathbf{G}$, run extremely close together.

The mode interaction as found in our pendulum system is analogous to the experimentally observed mode interaction in for instance a vibrating bowl of water 
$[9,10]$ and a driven double pendulum [3,4]. Indeed, our bifurcation lines $\mathbf{R}_{\mathbf{I} \text {, right }}-\mathbf{A}_{\text {III, high }}$ and $\mathbf{G}$ can also be recognized in these systems. In the fluid system these lines are called $c$ and $e$ by Crawford et al. (see Ref. [1] Fig. 7), and in the double pendulum system $s_{\theta}$ and $s_{\phi}$ (see Ref. [3] Fig. 2). The reported mixed modes are to be compared with both the MP- and the A-motion. However, a detailed comparison is not opportune yet since, near the mode interaction point, our (linearly coupled) system has a higher degree of degeneracy than the double pendulum and the vibrating bowl of water. This degeneracy can be lifted by the inclusion of additional nonlinearities in our system. In a forthcoming paper we shall see that an extra, non-linear term in the coupling between the pendulums already works wonders [8].

On a somewhat higher level of abstraction, however, the mode interaction takes place in much the same way in all three systems. That is, the group theoretical structure of our problem is the same as that of the double pendulum, and very similar to that of the fluid experiment. This will be discussed in a separate paper [5].

\section{Acknowledgements}

We would like to thank M. Oosterhof and J.T. Padding for a fruitful discussion about the dissipation threshold.

\section{Appendix A. The dissipation threshold}

In this appendix we will take a closer look at the dissipation threshold for a (symmetrical) one-pendulum motion, also applicable to the symmetrical 1-motions of our coupled pendulums. First, we will establish an approximation for small amplitudes of the pendulum; subsequently we will determine the limiting behavior for small $\Omega$. We will do so by considering the work balance between driving and dissipation (leaving out, as in our original equations of motion, a common factor $m \cdot l$ ). It may be noted that an analytical approximation for the dissipation threshold could also be obtained by applying Melnikov's methods to the exact solution of the undamped, undriven pendulum, as in excercise 4.6 .3 of Ref. [12].

The energy dissipated during one period of a (symmetrical one-pendulum oscillation is calculated by integrating the work done by the damping force over the period of the motion. As the damping is linear, it is easy to show that for a single pendulum performing a symmetrical $2 T$-oscillation this work is given by

$$
D=\int_{0}^{2 T} F_{d}(t) d \vartheta=\int_{0}^{2 T} \gamma \dot{\vartheta} \cdot \frac{d \vartheta}{d t} d t=\gamma \int_{0}^{2 T} \dot{\vartheta}^{2} d t,
$$

Let us first consider the harmonic approximation, in the understanding that this will only be valid for small amplitudes. At the threshold, where the $\alpha$ motion is 
annihilated together with its $\beta$ counterpart, the pendulum is observed to lag behind the forcing by about one quarter of the driving period. Thus we set:

$$
\vartheta(t)=\theta\left\{\cos \frac{1}{2} \Omega\left(t+\frac{1}{4} T\right)+\zeta \sin \frac{1}{2} \Omega\left(t+\frac{1}{4} T\right)\right\},
$$

where the sine term allows for a possible deviation of the phase lag from $\frac{1}{4} T$. This leads to

$$
D_{\text {harm }}=\gamma \int_{0}^{2 T} \dot{\vartheta}^{2} d t=\left(1+\zeta^{2}\right) \frac{\pi \gamma \Omega \theta^{2}}{2} .
$$

On the other side of the work balance, we have to calculate the work done on the pendulum by the drive mechanism. This is given by

$$
\begin{aligned}
W & =-\oint \frac{A \Omega^{2}}{l} \cos \Omega t \sin \vartheta d \vartheta \\
& =-\frac{A \Omega^{2}}{l} \int_{0}^{4 \pi / \Omega} \cos \Omega t \sin \vartheta(t) \cdot \dot{\vartheta}(t) d t .
\end{aligned}
$$

Using the harmonic approximation $\sin \vartheta \approx \vartheta$ we find

$$
W_{\mathrm{harm}}=-\frac{A \Omega^{2}}{l} \int_{0}^{4 \pi / \Omega} \cos \Omega t \vartheta \dot{\vartheta} d t=\left(1-\zeta^{2}\right) \frac{A \Omega^{2} \pi \theta^{2}}{2 l} .
$$

Equating the two expressions for $W_{\text {harm }}$ and $D_{\text {harm }}$ gives us the desired threshold value for $A$, for small motion amplitudes,

$$
\frac{A}{l}=\frac{1+\zeta^{2}}{1-\zeta^{2}} \cdot \frac{\gamma}{\Omega}
$$

This expressions shows that for small values of the amplitude of the motion the threshold value of $A / l$ behaves as $1 / \Omega$, in agreement with the loose argument presented in Section 1.2.

In Fig. A1 we have drawn the line given by Eq. (A.6) for several values of $\zeta$, together with the resonance tongue (given by Eq. (2.2) in the main text, and also in Appendix B). For $\zeta=0$ the dissipation threshold joins smoothly with the tongue, while for any $\zeta \neq 0$ the line given by Eq. (A.6) intersects the border of the tongue twice, which would imply a (quite unphysical) discontinuity in the slope of the threshold. Thus, we conclude that $\zeta=0$, that is, the phase difference between the driving and the pendulum motion is exactly $\frac{1}{4} T$ in the harmonic regime ${ }^{7}$. It also means that $\theta$ can be identified as the actual amplitude of the oscillation.

\footnotetext{
${ }^{7}$ Remarkably, without performing any bifurcation analysis, we have determined the point on the border of the resonance tongue where the transition from super- to subcritical- (i.e. from $\alpha$ to $\beta$ ) bifurcation takes place.
} 


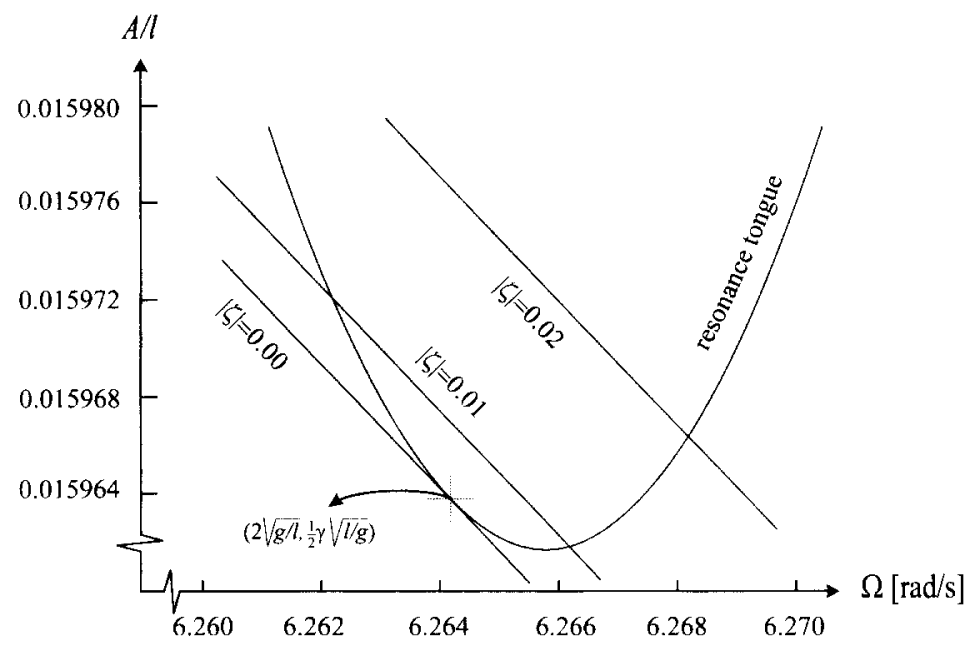

Fig. A1. The curve given by Eq. (A.6), for three different values of $\zeta$, together with the resonance tongue given by Eq. (2.2) in the main text. Only the line with $\zeta=0$ qualifies as the true threshold (in the harmonic approximation). The value of $\gamma$ was taken to be $0.1 \mathrm{~s}^{-1}$.

For low values of $\Omega$ the motion is of course far from harmonic and the above approximation fails. For these values of $\Omega$ we can however establish a lower limit for the threshold, by overestimating the amount of energy pumped into the system by the driving and underestimating the amount of energy lost to dissipation. In Fig. A2a we have depicted the $\cos \Omega t$ and $\sin \vartheta \dot{\vartheta}(t)$ terms, which appear in the expression for $W$ The figure shows that the phase difference between the driving force and the pendulum's oscillation is apparently still $\frac{1}{4} T$ at these large amplitudes and one can infer that the desired upper limit for $W$ is obtained by substituting a block-function for the driving term, giving

$$
W_{\max }=-2 \frac{A \Omega^{2}}{l} \int_{-\vartheta_{\max }}^{\vartheta_{\max }}|\sin \vartheta| d \vartheta=\frac{4 A \Omega^{2}\left(1-\cos \vartheta_{\max }\right)}{l}
$$

Now let us find a lower bound for the amount of energy lost due to dissipation. Taking $D_{\min }=0$ would be trivial, but we can do better than that. Consider Fig. A2b, displaying the projection of a typical trajectory of the pendulum onto the $(\vartheta, \dot{\vartheta})$-plane. Eq. (A.1) tells us that the dissipation integral equals $\gamma$ times the area covered by the solid curve. The shape of this curve is something between a diamond (with area $2 \vartheta_{\max } \dot{\vartheta}_{\max }$ ) and an ellipse (with area $\pi \vartheta_{\max } \dot{\vartheta}_{\max }$ ); the diamond may be used to get the desired underestimate. Along the threshold $\vartheta_{\max }$ and $\dot{\vartheta}_{\max }$ vary as a function of $\Omega$, and we may put

$$
\dot{\vartheta}_{\max }(\Omega)=h(\Omega) \vartheta_{\max }(\Omega)
$$




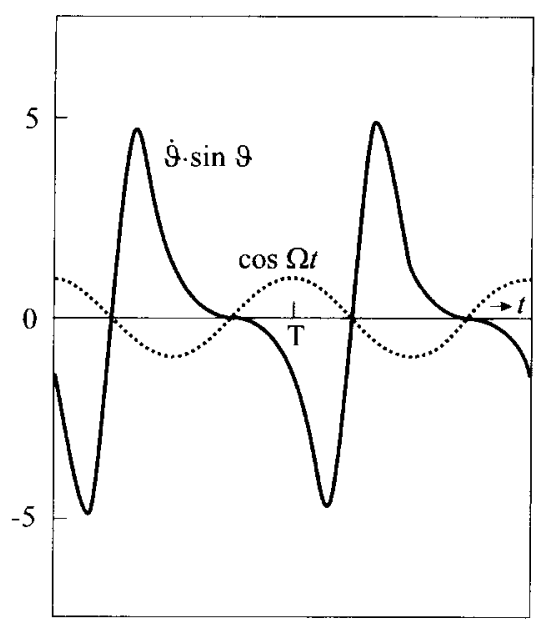

(a)

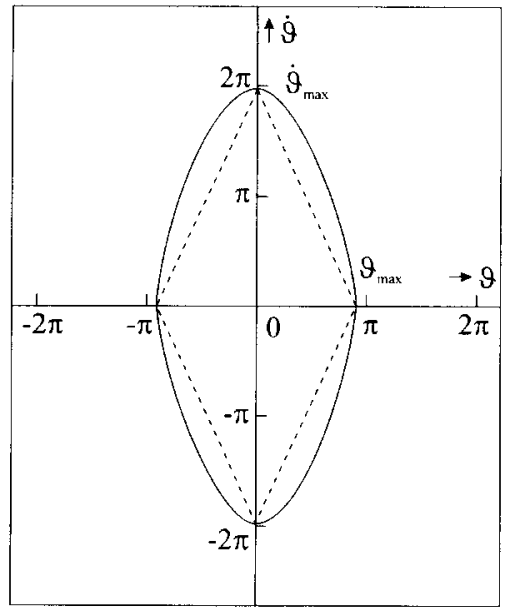

(b)

Fig. A2. Numerical data for one pendulum (or equivalently for the 1-motions) for $\Omega=2.756 \mathrm{rad} / \mathrm{s}$, $A / l=0.123, \gamma=0.1 \mathrm{~s}^{-1}$; (a) the curves $\cos \Omega t$ and $\sin \vartheta \cdot \dot{\vartheta}(t) ;(b)$ projection onto the $(\vartheta, \dot{\vartheta})$-plane.

For the minimum value of the dissipation integral (corresponding with the diamond) we then find

$$
D_{\min }=2 \gamma h(\Omega) \vartheta_{\max }^{2}(\Omega)
$$

Equating Eqs. (A.7) and (A.9), i.e., setting $W_{\max }=D_{\min }$, provides us with a curve that lies below the real threshold

$$
\frac{A}{l}=\frac{2 \gamma h(\Omega) \vartheta_{\max }^{2}(\Omega)}{4 \Omega^{2}\left(1-\cos \vartheta_{\max }(\Omega)\right)}
$$

We would like to know the dependence of $\vartheta_{\max }$ and $h$ on $\Omega$, in order to express the lower bound for the threshold solely in terms of $\Omega$. Now, the amplitude of a pendulum oscillation (and of the symmetrical 1-motion in the two-pendulum system) is never seen to go beyond $\pi$, just as in the absence of driving, and approaches this value when we decrease $\Omega$. This is corroborated by Fig. A3, where we have plotted the numerically obtained amplitude $\vartheta_{\max }$ as a function of $\Omega$. In the same figure the function $h(\Omega)$ is given, which is seen to approach a value close to 2 for sufficiently small $\Omega$. It is intriguing that this same value would also be found for a non-driven, non-dissipated pendulum. In that case energy conservation gives the following relation between $\dot{\vartheta}_{\max }$ and $\vartheta_{\max }$ :

$$
\dot{\vartheta}_{\max }=2 \sqrt{g / l} \sin \left(\frac{1}{2} \vartheta_{\max }\right)
$$




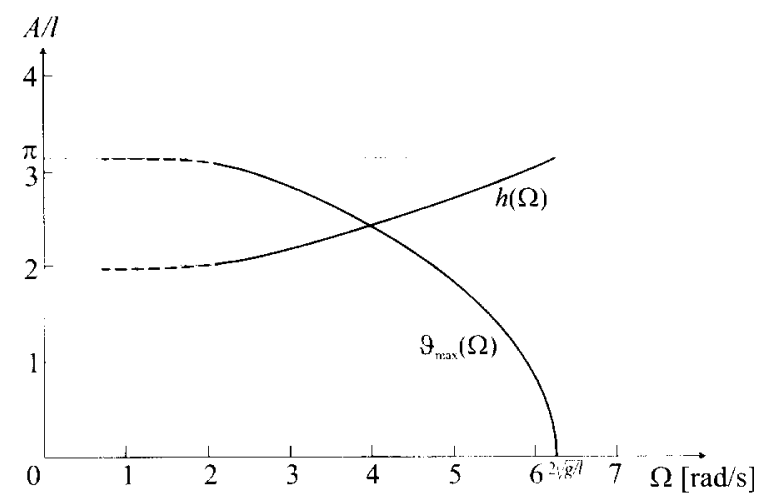

Fig. A3. Numerical data for $\vartheta_{\max }(\Omega)$ and $h(\Omega)$ on the threshold. For sufficiently small $\Omega$ these functions are seen to approach $\pi$ and (a value close to) 2 , respectively.

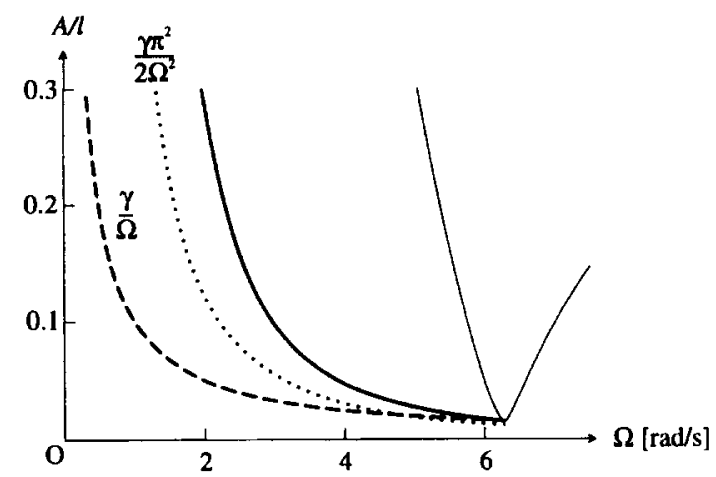

Fig. A4. The threshold line $\mathbf{T}_{1}$ for the symmetrical 1-motion (solid), together with its lower limit (dotted). and its harmonic estimate (dashed).

For $\vartheta_{\max } \approx \pi$ we find $\dot{\vartheta}_{\max } \approx 2 \sqrt{g / l}=6.26$, which happens to be approximately equal to $2 \pi \approx 2 \vartheta_{\max }$. So, for the non-driven pendulum without dissipation we also have $h \approx 2$ for amplitudes close to $\pi$.

Putting $\vartheta_{\max }=\pi$ and $h(\Omega)=2$ into Eq. (A.10) gives

$$
\frac{A}{l}=\frac{\gamma \pi^{2}}{2 \Omega^{2}} .
$$

We have drawn the curves given by Eqs. (A.6) (dashed) and (A.12) (dotted) in Fig. A4. The solid curve represents the real, numerically obtained threshold for the symmetrical 1-motion. From this figure it is clear that the harmonic estimate, given by Eq. (A.6), is indeed only valid for frequencies close to the birthline of the 1-motion, i.e., for very small motion amplitudes. We also see that Eq. (A.12) is indeed a lower bound for the threshold for sufficiently small values of $\Omega$. 


\section{Appendix B. The resonance tongues}

In this appendix we will derive the expressions (2.2) and (2.3) in the main text, for the borders of the region of stability for the 0 -motion. The method closely resembles the one outlined by Landau and Lifschitz in Ref. [13].

The equation of motion governing stability of the 0 -motion with respect to perturbations in the 1-direction is given by

$$
\ddot{\phi}_{1}+\left(\frac{g}{l}+\frac{A \Omega^{2}}{l} \cos \Omega t\right) \phi_{1}+\gamma \dot{\phi}_{1}=0 .
$$

In the absence of driving and dissipation, the solution of this equation of motion would be

$$
\phi_{1}=a \cos \omega t+b \sin \omega t
$$

Therefore, in first approximation, one may assume that the solution to Eq. (B.1) is of the form

$$
\phi_{1}=a(t) \cos \omega t+b(t) \sin \omega t \quad \text { with } \omega=\frac{1}{2} \Omega
$$

with $a(t)$ and $b(t)$ varying in time sufficiently slow, so that $\ddot{a}(t) \approx 0$ and $\ddot{b}(t) \approx 0$. The exact solution to Eq. (B.1) will of course include higher harmonics $(3 \omega, 5 \omega$, etc.), but they are of little importance ${ }^{8}$. Substituting Eq. (B.3) into Eq. (B.1), and using the approximations just stated, we find that

$$
\begin{aligned}
& \cos \omega t\left(-a \omega^{2}+2 \dot{b} \omega+\gamma \dot{a}+\gamma b \omega+\frac{g}{l} a-\frac{2 A \omega^{2}}{l} a\right) \\
& +\sin \omega t\left(-2 \dot{a} \omega-b \omega^{2}+\gamma \dot{b}-\gamma a \omega+\frac{g}{l} b+\frac{2 A \omega^{2}}{l} b\right)=0 .
\end{aligned}
$$

This equation must hold for all $t$, and hence the expressions within parentheses are both identically zero. Putting this in matrix form one gets:

$$
\left(\begin{array}{cc}
\gamma & 2 \omega \\
-2 \omega & \gamma
\end{array}\right)\left(\begin{array}{l}
\dot{a} \\
\dot{b}
\end{array}\right)=\left(\begin{array}{cc}
\omega^{2}-\frac{g}{l}+\frac{2 A \omega^{2}}{l} & -\gamma \omega \\
\gamma \omega & \omega^{2}-\frac{g}{l}-\frac{2 A \omega^{2}}{l}
\end{array}\right)\left(\begin{array}{l}
a \\
b
\end{array}\right)
$$

\footnotetext{
${ }^{8}$ This approximation is valid in the region we are most interested in (which includes the intersection point of the two resonance tongues). For $A / l>0.2$, however, the borders of the tongues evaluated with this approximation begin to deviate discernibly from the numerically obtained ones.
} 
Writing $a(t)=a e^{s t}$ and $b(t)=b e^{s t}$, this yields the following eigenvalue equation:

$$
\left(\begin{array}{cc}
\gamma & 2 \omega \\
-2 \omega & \gamma
\end{array}\right)^{-1}\left(\begin{array}{cc}
\omega^{2}-\frac{g}{l}+\frac{2 A \omega^{2}}{l} & -\gamma \omega \\
\gamma \omega & \omega^{2}-\frac{g}{l}-\frac{2 A \omega^{2}}{l}
\end{array}\right)\left(\begin{array}{l}
a \\
b
\end{array}\right)=s\left(\begin{array}{l}
a \\
b
\end{array}\right)
$$

Evidently, the proposed solution (B.3) will explode when the eigenvalue $s$ has a positive real part, and die out when its real part is negative. The trivial solution to Eq. (B.1) (that is, the 0-motion) is therefore unstable when

$$
\operatorname{Re}(s)>0 \text {. }
$$

A short calculation (remembering that $2 \omega=\Omega$ ) transforms (B.7) into

$$
\frac{A}{l}>\frac{1}{2} \sqrt{\left(1-4 \frac{g / l}{\Omega^{2}}\right)^{2}+4 \frac{\gamma^{2}}{\Omega^{2}}}
$$

which is Eq. (2.2) in the main text. Analogously, we find that the 0-motion is unstable with respect to perturbations in the 2-direction if

$$
\frac{A}{l}>\frac{1}{2} \sqrt{\left(1-4 \frac{g / l+2 K}{\Omega^{2}}\right)^{2}+4 \frac{\gamma^{2}}{\Omega^{2}}} .
$$

This is Eq. (2.3) in the main text.

It follows from Eqs. (B.8) and (B.9) that the lowest points of the two tongues are given by

$$
\Omega=\frac{2 \sqrt{g / l}}{\sqrt{1-\gamma^{2} / 2 g / l}}, \quad \frac{A}{l}=\frac{\gamma}{2 \sqrt{g / l}} \sqrt{1-\frac{\gamma^{2}}{4 g / l}},
$$

and

$$
\Omega=\frac{2 \sqrt{g / l+2 K}}{\sqrt{1-\gamma^{2} / 2(g / l+2 K)}}, \quad \frac{A}{l}=\frac{\gamma}{2 \sqrt{g / l+2 K}} \sqrt{1-\frac{\gamma^{2}}{4(g / l+2 K)}},
$$

respectively, which shows that the tip of the right tongue lies lower than that of the left tongue (see also Fig. B1). Moreover, both minima have (slightly) been shifted to the right with respect to their Hamiltonian positions. The intersection point of the two tongues is given by

$$
\Omega=2 \sqrt{g / l+K}, \quad \frac{A}{l}=\frac{1}{2} \sqrt{\left(1-\frac{g / l}{g / l+K}\right)^{2}+\frac{\gamma^{2}}{g / l+K}} .
$$

So we see that, although dissipation shifts the minima of the two tongues to the right, the $\Omega$-coordinate of the intersection point remains unchanged. 


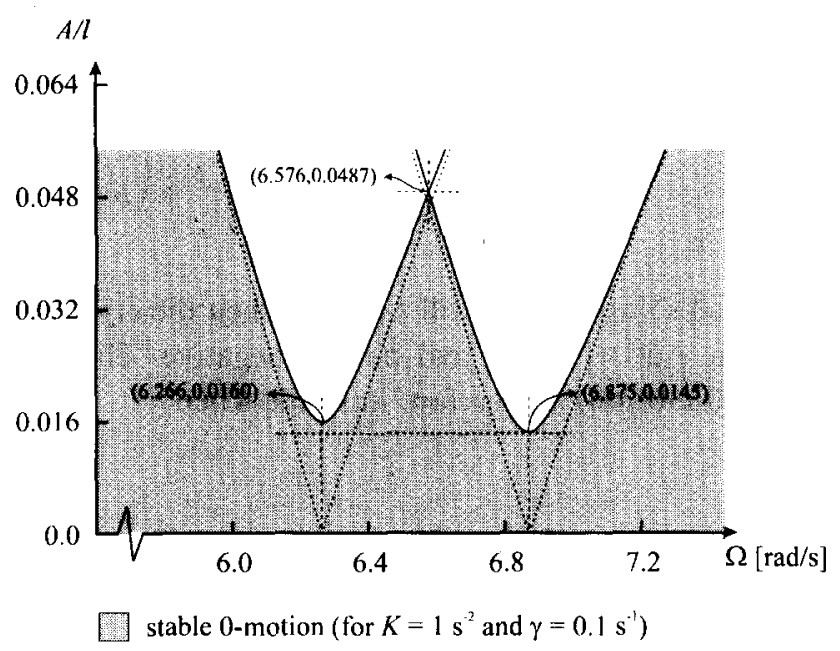

Fig. B1. The lower part of the first-order resonance tongues.

\section{References}

[1] E.J. Banning, J.P. van der Weele, Mode competition in a system of two parametrically driven pendulums; the Hamiltonian case, Physica A 220 (1995) 485-533.

[2] P.T. Squire, Pendulum damping, Amer J. Phys. 54 (1986) $984-991$.

[3] A.C. Skeldon, T. Mullin, Mode interaction in a double pendulum, Phys. Lett. A 166 (1992) 224-229.

[4] A.C. Skeldon, Dynamics of a parametrically excited double pendulum, Physica D 75 (1994) 541- 558.

[5] E.J. Banning, J.P. van der Weele, M.M. Kettenis, Mode competition in a system of two parametrically driven pendulums: the role of symmetry, Physica A, in press (1997).

[6] J.E. Howard, R.S. MacKay, Linear stability of symplectic maps, J. Math. Phys. 28 (1987) 1036-1051.

[7] Th. Zeegers, On the existence of infinite period-doubling sequences in a class of 4D semi-symplectic mappings, J. Phys. A: Math. Gen. 24 (1991) 2287-2314.

[8] E.J. Banning, J.P. van der Weele, J.C. Ross, M.M. Kettenis, Mode competition in a system of two parametrically driven pendulums with nonlinear coupling, Physica A 245 (1997) 49-98, this issue.

[9] S. Ciliberto, J.P. Gollub, Pattern competition leads to chaos, Phys. Rev. Lett. 52 (1984) 922-925.

[10] S. Ciliberto, J.P. Gollub, Chaotic mode competition in parametrically forced surface waves, J. Fluid Mech. 158 (1985) 381-398.

[11] J.D. Crawford, E. Knobloch, H. Riecke, Period-doubling mode interactions with circular symmetry, Physica D 44 (1990) 340-396.

[12] J. Gucksheimer, P. Holmes, Nonlinear oscillations, Dynamical Systems, and Bifurcations of Vector Fields, in: Applied Mathematical Sciences, Vol. 42, Springer, New York, 1983.

[13] L.D. Landau, E.M. Lifschitz, Mechanics, Vol. 1 of Course of Theoretical Physics, Pergamon Press, Oxford, 1960, pp. 80-83. 